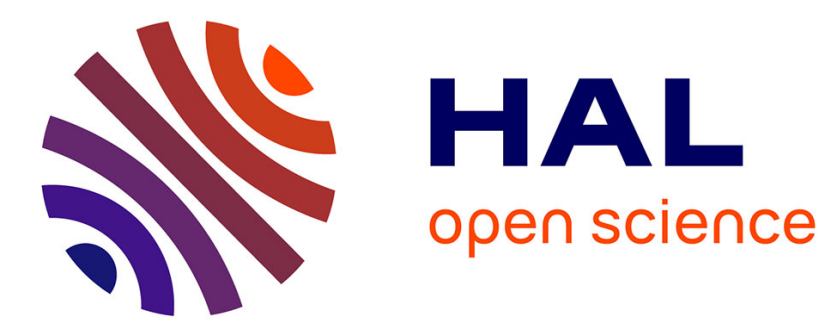

\title{
Modeling fretting-corrosion wear of 316L SS against poly(methyl methacrylate) with the Point Defect Model: Fundamental theory, assessment, and outlook Jean Geringer, Digby D. Macdonald
}

\section{- To cite this version:}

Jean Geringer, Digby D. Macdonald. Modeling fretting-corrosion wear of 316L SS against poly(methyl methacrylate) with the Point Defect Model: Fundamental theory, assessment, and outlook. Electrochimica Acta, 2012, 79, pp.17-30. 10.1016/j.electacta.2012.06.028 . hal-00753542

\author{
HAL Id: hal-00753542 \\ https://hal.science/hal-00753542
}

Submitted on 19 Nov 2012

HAL is a multi-disciplinary open access archive for the deposit and dissemination of scientific research documents, whether they are published or not. The documents may come from teaching and research institutions in France or abroad, or from public or private research centers.
L'archive ouverte pluridisciplinaire HAL, est destinée au dépôt et à la diffusion de documents scientifiques de niveau recherche, publiés ou non, émanant des établissements d'enseignement et de recherche français ou étrangers, des laboratoires publics ou privés. 


\title{
MODELING FRETTING-CORROSION WEAR OF 316L SS AGAINST POLY(METHYL METHACRYLATE) WITH THE POINT DEFECT MODEL: FUNDAMENTAL THEORY, ASSESSMENT, AND OUTLOOK
}

\author{
$\underline{\text { J. Geringer }}^{\mathrm{a}, \mathrm{b}, 1,{ }^{*}}$, D. D. Macdonald ${ }^{\mathrm{b}, \mathrm{c}, 1}$ \\ ${ }^{a}$ Ecole Nationale Supérieure des Mines de Saint-Etienne, ENSM-SE, \\ Center for Health Engineering, UMR CNRS 5146, IFR 143 \\ Department of Biomechanics and Biomaterials-Bio-tribocorrosion, \\ 158 cours Fauriel, F-42023 Saint-Etienne Cedex 02 \\ Tel: +(33).477426 688; Fax: +(33).477429 755; geringer@emse.fr; jgeringer@voila.fr \\ ${ }^{b}$ Center for Electrochemical Science and Technology, \\ Department of Materials Science and Engineering \\ Pennsylvania State University, University Park, PA 16802, USA \\ Tel: +(1) 814863 7772; Fax: +(1) 814863 4718; ddm2@psu.edu \\ ${ }^{c}$ Center of Research Excellence in Corrosion \\ Research Institute \\ King Fahd University of Petroleum and Minerals \\ Dhahran, Saudi Arabia \\ Tel: +(966)038607 551,ddm2@psu.edu
}

\begin{abstract}
This work aims at investigating experimentally fretting corrosion, that is, corrosion induced by friction of AISI 316L SS against poly(methyl methacrylate) under small displacements, and subsequently using the data to model wear. The observed wear on the stainless steel has been modeled using the Point Defect Model (PDM). The originality of this approach in applying the PDM to fretting corrosion consists of using a modified rate of the barrier layer dissolution, in the case of cyclical wear, such that the rate of destruction of the barrier layer at the barrier layer/solution interface exceeds the rate of barrier layer growth at the metal/barrier layer interface at zero barrier layer thickness, which is the condition specified by the PDM for depassivation. By optimization of the PDM on experimental electrochemical impedance data under fretting conditions, we have been able to ascertain values for various model parameters, and to calculate the steel volume loss as a function of potential.
\end{abstract}

Keywords: Point Defect Model; Tribo-corrosion; Fretting corrosion; Wear, Stainless Steel.

*: Corresponding author

${ }^{1}$ ISE Member 


\section{1- Introduction}

Fretting corrosion, which arises from friction between two surfaces under small displacement loading conditions in a corrosive medium, is related to the stability of the passive film on the metal or alloy surface. As we show in this paper, the passivity of an alloy is susceptible to fretting, because mechanical friction may destroy the barrier oxide layer more rapidly than the barrier layer can grow into the metal at zero barrier layer thickness, thereby inducing depassivation [1]. It is probable that the same process of degradation occurs in erosion-corrosion and particle impact corrosion. However the difference between fretting corrosion and particle impact corrosion lies in the properties of the particles impacting the surface of the alloy. The key points about the wastage rate of particle impact corrosion are particles size (mass), particle flux, $\mathrm{pH}$ of the solution, etc. [1], while the important independent variables for fretting corrosion include contact pressure, displacement, relative velocity of the two surfaces and the existence of a crevice, with the external chemical properties often being of secondary importance.

In the case of fretting corrosion, modeling the degradation of the alloy in terms of the stability of the passive film is a complex task, involving a large number of variables describing a system of great physico-chemical complexity. By carefully combining experimental studies with modeling, it is possible to describe the physico-electrochemical processes involved in fretting and to predict the wear rate, i.e. the wastage rate [2]. A common strategy in the case of fretting between an insulating material and a metallic alloys is to interpret fretting corrosion as an accelerated form of corrosion due to wear. However, the usual galvanic series that is often used under non-fretting conditions to rationalize corrosion phenomens between dissimilar metals in close proximity and in ohmic contact, is considerably modified by the impact of the mechanical degradation of the passive film. Moreover, synergistic effects between various parameters and processes are significant and must be recognized, in order to develop a satisfactory explanation of the overall fretting degradation phenomenon. Sometimes iterative, numerical methods are necessary for modeling the combination of mechanical- and corrosive- degradation, because of the complexity of the constitutive, non-linear equations that often do not have analytical solutions [3]. In this article, we adopt the same strategy by combining experimental studies with a model for the passive state that was developed to describe at the atomic scale the processes that lead to the growth and breakdown of the passive film. The resulting model is optimized on the experimental data to determine values for poorly known model parameters. The optimized model is then used to predict the accumulation of damage beyond the range of optimization.

Fretting corrosion is one of the principal topics of study in the orthopedic-implant field [4-11]. It is worth noting that this phenomenon also plays a role in the accumulation of corrosion damage in a wide range of systems, including nuclear power plants, where contacting surfaces are often in relative motion 
[12]. The present study is focused on hip implants and especially on the contact of Type 316L stainless steel against bone cement, which is modeled by poly(methyl methacrylate), PMMA. Hip implants are commonly manufactured from Type 316L SS, because of its good toughness, strength, and corrosion resistance. In its clinical use, the steel stem is inserted into the femoral bone and then fixed with bone cement, consisting principally of PMMA. In the human body, the stainless steel is immersed in a physiological solution, having a high chloride concentration $(\sim 0.2 \mathrm{M})$ at a temperature of $38{ }^{\circ} \mathrm{C}$. Because of the significant difference of mechanical properties (especially Young's modulus, a ratio of 100 between 316L and PMMA), stress shielding occurs. This physical phenomenon can be explained as following. Metallic materials and the polymers do not exhibit the same strain under applied stress, thus leading to disbonding between the metal and the cement. Accordingly, adhesion between the metal and the polymer is lost after a short time of human gait. This debonding involves fretting (friction under small displacements, a term that is defined precisely later in this paper) and subsequent friction between the materials in contact. Thereafter, the materials (stainless steel and bone cement) are subjected to small displacement, frictional loading, in a corrosive environment, resulting in fretting corrosion. Consequently, wear occurs between the bone cement and stainless steel; it involves debris generation (corrosion products) that often produces inflammation reactions, concluding with aseptic loosening of the implants and the need for replacement [5].

Passivity breakdown on stainless steels is a topic that has been subjected to numerous experimental and theoretical studies. In this paper, the authors have chosen to investigate the progression of damage from the perspective of the Point Defect Model (PDM) [13-21]. It is worth noting that, in order to use the PDM, values for many important physical parameters have been extracted from the literature, or have been determined experimentally (oxides layer thickness, for instance). Parameter values were selected, to the greatest extent possible, so as to minimize any differences that may exist between the experimental conditions and those assumed in the simulation.

Modeling of fretting corrosion damage using the PDM is shown to be capable of accurately predicting wear. The principal goal is the prediction of the particular ' $\mathrm{W}$ ' wear-shaped area observed during fretting corrosion between Type 316L SS and PMMA [22]. The oxide layer thickness and the current density are derived as a function of $\mathrm{pH}$ and applied potential under steady state conditions without and with fretting. Finally, the fretting contribution is defined, and the wear depth profiles are obtained using the customized PDM, and they are compared with actual wear depth profiles observed experimentally. The oxide film thickness and the current density depend strongly on the local potential, because of the $I R$ potential drop, and on the local $p H$ in the wear track area. These dependencies are examined, in particular, because of their influence on the wear profile and hence on the development of damage. The results are also discussed in terms of crevice corrosion within the friction zone. 


\section{Experimental Techniques}

The experimental apparatus and techniques are described in a previous paper [22]. Experiments to understand the development of fretting corrosion were carried out with contact between AISI Type 316L SS and PMMA, under well-controlled electrochemical conditions. The PMMA is a convenient material for these studies, because it is transparent, thereby allowing direct observation of the fretting zone. Consequently, the progression of the corrosive degradation of the steel has been recorded microscopically in "real time". The fretting corrosion device and tests conditions, i.e. normal load and displacement, were selected to be close to the ones that occur in the human body in practice, and have been described in Ref. [22]. The corrosive, physiological medium is Ringer's solution with a high chloride concentration of around $0.2 \mathrm{M}$. The influence of organic material (e.g., proteins) on the corrosion process is not considered. Experiments have been carried out at the open circuit (corrosion) potential to highlight dissolution and wear of 316L SS that could occur in the human body. Moreover, experiments have also been carried out under controlled potential conditions, in which the current density between the working electrode, Type 316L SS, and the counter electrode made of platinum, was monitored. The reference electrode was a Saturated Calomel Electrode, SCE. Post test analyses included SEM and 3D mechanical profilometry, in order to show, qualitatively and quantitatively, the profile of the wear track area. Current density, wear volume, and wear depth, including those reported in a previous study [22], were measured, in order to compare the damage with that predicted by the Point Defect Model, PDM.

\section{Results and Discussion}

\subsection{Principal Experimental Results on Fretting Corrosion}

First, it is worth noting that oxide spalling and settlement in the fretting experiment occur primarily at the rim of the contact zone. Consequently, one might expect that the $\mathrm{pH}$ inside the contact zone, would be very low, e.g. lower than 1. Indeed, according to the Pourbaix diagrams for $\mathrm{Cr}$ and $\mathrm{Fe}$ [23], chromium and iron oxides do not precipitate under $p H=1$ conditions, although they may exist as meta-stable phases that form directly from the metal (e.g., as the barrier layer of a passive film) [24]. The experimental results demonstrate that chromium oxides deposit near the contact rim and that iron oxides are found at $1.7 \mathrm{~mm}$ from the contact rim [25]. These results may indicate that a $p H$ gradient exists from the wear track area to the outside, as expected from crevice corrosion theory. All results presented in the following part of this paper were originally presented in Refs. [22, 26] and relevant information has been extracted therefrom for this analysis. 
Figure 1 shows 2D and 3D images of wear zones on Type 316L SS samples, as created by fretting corrosion on contact with PMMA in Ringer's solution, at different applied potentials. The 2D profiles exhibit a particular ' $\mathrm{W}$ ' wear shape, as noted previously (Geringer et al. [22]). Wear is higher at 0 $\mathrm{mV}(\mathrm{SCE})$ than at $-200 \mathrm{mV}(\mathrm{SCE})$. This wear shape has been obtained at all applied potentials. One might be tempted to explain the wear shape in terms of the ohmic potential drop and the $p H$ change, as has been articulated for crevice corrosion by Pickering et.al. [27-32], but, as we shall see, fretting (the relative motion of two surfaces in intimate contact) is an essential component of the development of damage. Moreover, evolution of the local potential may also account for promoting metal dissolution inside the contact zone and at the rim. The PDM has been modified to take into account these additional processes.
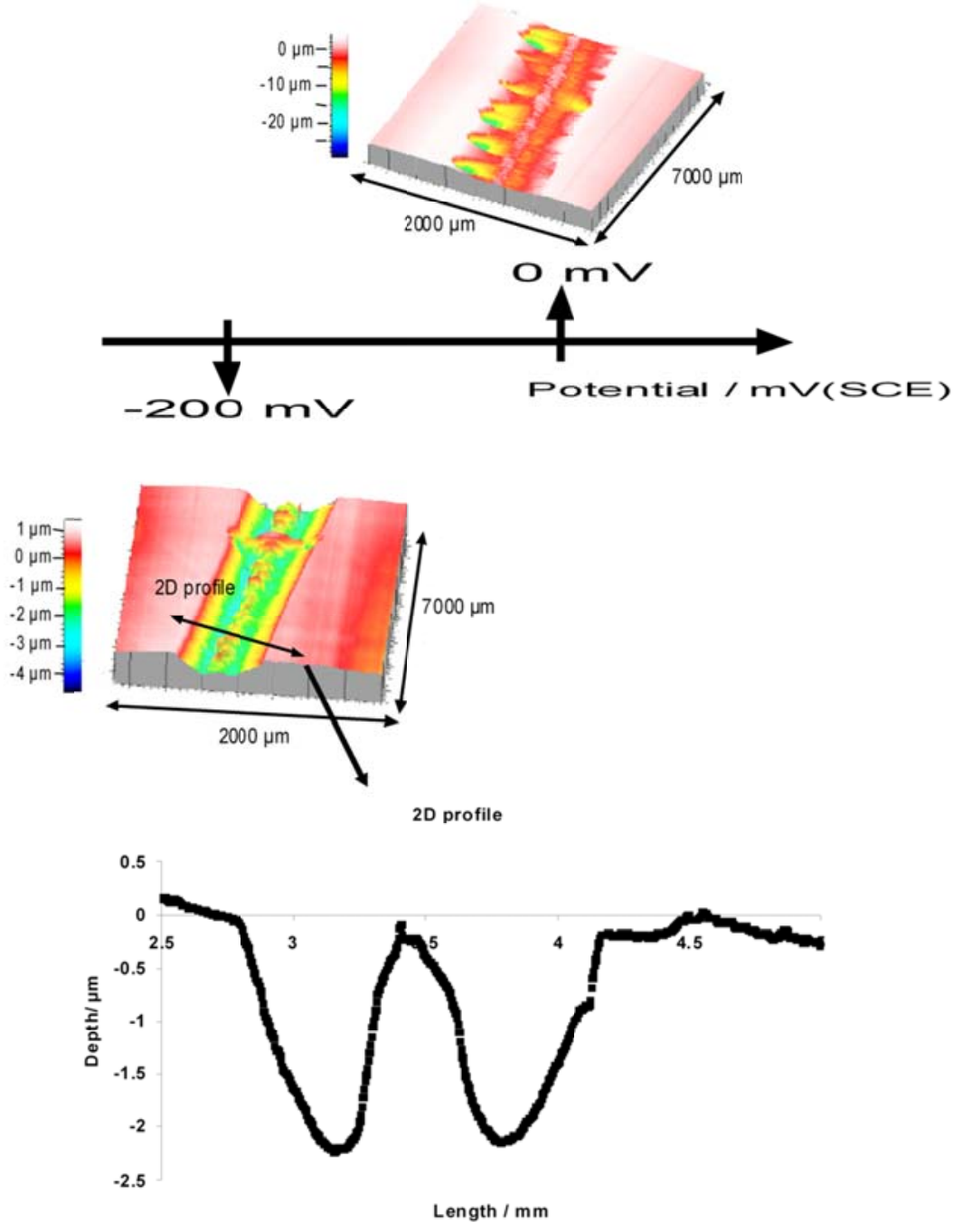

Figure 1: 2D image and 3D images of worn samples of Type 316L SS at applied potentials of 0 and $200 \mathrm{mV}$ (SCE) after fretting corrosion for 20,000 cycles. 
Figure 2 displays curves of imposed displacement, tangential load, and current during one second of fretting, at $-200 \mathrm{mV}$ (SCE) and after 20,000 seconds of continuous fretting. The current varies with the rate of change of the amplitude. This experimental result demonstrates that the measured current is related to the relative displacement of the Type 316L SS and the PMMA surfaces. When the two surfaces are in relative motion, the mechanical destruction of the passive layer results in an increase in the current density. It is worth noting that, when the displacement velocity decreases, the current density follows the same trend. This implies that the current density is related to friction-induced, energy dissipation $[22,26]$.

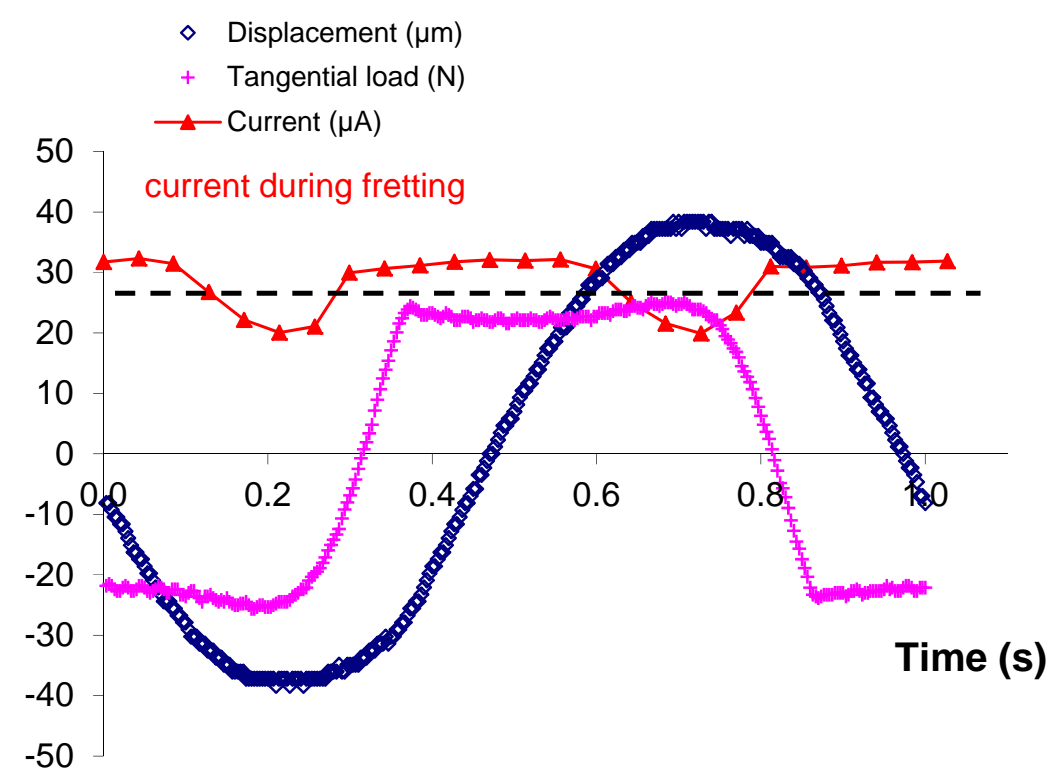

Figure 2: Imposed displacement $(\mu \mathrm{m})$, tangential load $(\mu \mathrm{m})$, and current $(\mu \mathrm{A})$ during 1 second of fretting at an applied potential of $-200 \mathrm{mV}$ (SCE) after 20,000 seconds of continuous fretting corrosion.

Table 1 exhibits the values of the current density, measured at different applied potentials, before and during fretting corrosion. The quantity, $\triangle$, which is the contribution to the current density from fretting, is the difference in the current density between when fretting exists and when it does not exist. The potential of $-400 \mathrm{mV}(\mathrm{SCE})$ corresponds to the potential at which the net current density just becomes anodic (positive) (Table 1). At this potential, without friction, the net current density is negative (preponderance of cathodic processes); with friction, the current density becomes positive, indicating that anodic reactions dominate the charge transfer behavior of the steel, with fretting enhancing the anodic partial current compared with the cathodic partial reactions (e.g., hydrogen evolution, oxygen reduction) in the mixed potential description of the system. 
Table 1: Current density before and during fretting at different applied potentials.

\begin{tabular}{cccc} 
Potential mV(SCE) & $\mathbf{- 4 0 0}$ & $\mathbf{- 2 0 0}$ & $\mathbf{0}$ \\
\hline Current density before fretting $\left(\mu \mathrm{A} \cdot \mathrm{cm}^{-2}\right)$ & $-3 \pm 2$ & $2.5 \pm 1$ & $44 \pm 2$ \\
Current density during fretting $\left(\mu \mathrm{A} \cdot \mathrm{cm}^{-2}\right)$ & $1 \pm 2$ & $13 \pm 4$ & $83 \pm 6$ \\
$\triangle$ current density during fretting $\left(\mu \mathrm{A} \cdot \mathrm{cm}^{-2}\right)$ & $3 \pm 1$ & $7 \pm 3$ & $22 \pm 3$
\end{tabular}

Concerning the use of the PDM, it is necessary to know the wear depths for known fretting times and applied potential. Indeed, the wear depth is inserted into the PDM as a direct measure of the extent of damage. Table 2 represents the time/wear coefficient (linear wear rate) vs. the applied potential for Type 316L SS for different fretting times. The results are extracted from the previous investigation [22]. Evidently, the time/wear coefficient increases as the applied potential moves in the positive direction, again demonstrating that fretting enhances the anodic partial process. Between -800 and $-600 \mathrm{mV}$ (SCE), no wear was measurable. Type 316L SS exhibits no significant degradation by fretting against PMMA within this range of potential. Apparently, the barrier layer in unaffected, and does not lose its protective properties, within this potential range or that the alloy is "cathodically protected" $[-600$ to -800 $\mathrm{mV}(\mathrm{SCE})]$.

Thus, with invocation of Faraday's law, a comparison between the actual and calculated 2D profiles can be investigated. However, before that is done, the PDM will be optimized on wear data at fixed applied potentials, because this procedure is convenient for calculating the ohmic drop in the crevice between the two fretting surfaces. 
Table 2: Wear depth as a function of applied potential. The Wear Depth Coefficient is the slope of the straight line of wear depth vs. fretting duration.

\begin{tabular}{cccc} 
Potential $\mathrm{mV}(\mathrm{SCE})$ & Potential $\mathrm{mV}(\mathrm{SHE})$ & $\begin{array}{c}\text { Wear depth, wear-time } \\
\text { coefficient } \\
10^{-4} \mu \mathrm{m}^{-\mathrm{s}^{-1}}\end{array}$ & $\begin{array}{c}\text { Correlation } \\
\text { coefficient }\end{array}$ \\
\hline-1000 & -750 & 0.01 & $\mathrm{r}^{2}$ \\
-800 & -550 & No wear measurable & 0.94 \\
-600 & -350 & No wear measurable & \\
-400 & -150 & 0.40 & 0.99 \\
-200 & +50 & 1.09 & 0.97 \\
0 & +250 & 1.77 & 0.93 \\
Free corrosion & 0.62 & 0.62 & 0.93 \\
potential & & &
\end{tabular}

\section{2: The Point Defect Model}

According to the Point Defect Model (PDM), a passive film is envisaged to be a bi-layer structure comprising a point defective, nano-crystalline barrier layer and a porous outer layer that is formed by the hydrolysis of cations that are transmitted through the barrier layer and are subsequently precipitated as a hydroxide, oxyhydroxide, or oxide, depending upon the local conditions [13-21]. The stoichiometric formula for the barrier layer oxide on the surface of the stainless steel can be represented as $\mathrm{MO}_{\chi / 2}$, without specifying the exact defect structure. As noted elsewhere [13,14], this barrier layer oxide possesses high concentrations of point defects in the form of vacancies on the metal and oxygen sublattices and metal interstitials. Processes occurring at the two interfaces are emphasized, because it is at these locations that the defects are generated and/or annihilated. The interfaces are assumed to be not at equilibrium and hence the rates of film growth and destruction at the metal/barrier layer $(\mathrm{m} / \mathrm{bl})$ and the barrier layer/outer layer (bl/ol) interfaces, respectively, are described in terms of kinetic principles. The potential profile across the barrier layer is envisioned to be as illustrated in Figure 3. 


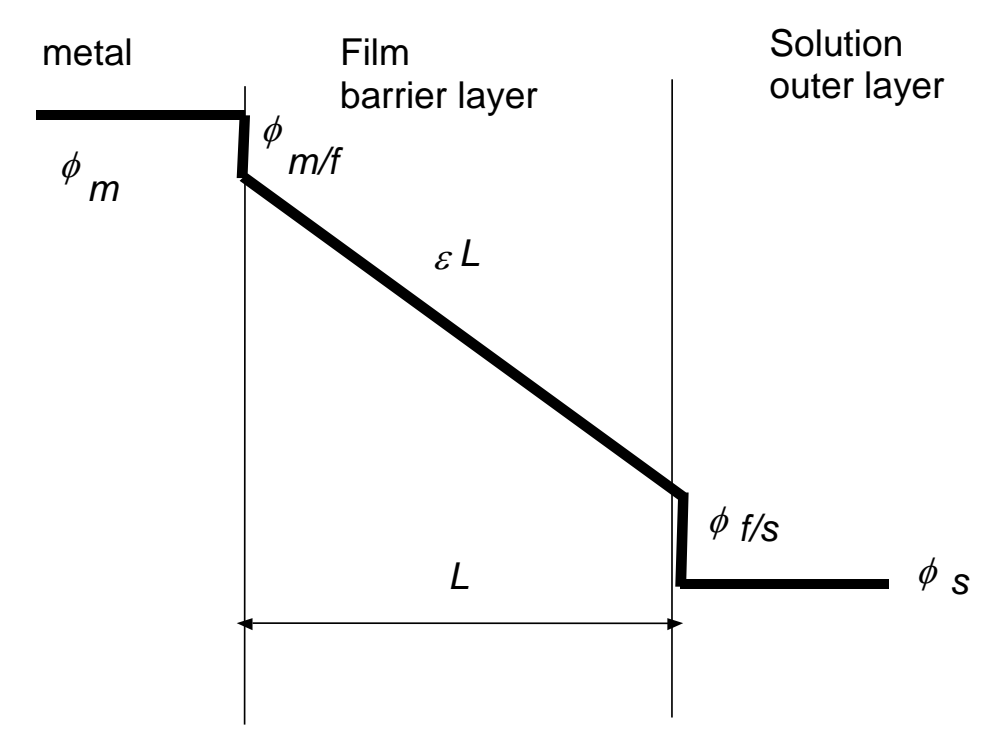

Figure 3: Schematic of the potential distribution across the barrier layer of a passive film.

The potential drop across the barrier layer/outer layer (bl/ol) interface, $\phi_{f / s}$ is assumed to be a linear function of the applied voltage and $\mathrm{pH}$ as [13-15]:

$\phi_{f / s}=\alpha V+\beta p H+\phi_{f / s}^{0}$

where $\alpha$ is the dependence of the potential drop across the bl/ol, interface on the applied voltage, and $\beta$ is the dependence of the potential drop across the same interface on the $p H$. The parameter $\phi_{f / s}^{\circ}$ is a constant (the value of $\phi_{f / s}$ at $V=0$ and $p H=0$ ). By noting that

$V=\phi_{m}-\phi_{1}+\phi_{1}-\phi_{2}+\phi_{2}-\phi_{s}$

(see Figure 3) and that $\phi_{m / f}=\phi_{m}-\phi_{1}, \phi_{1}-\phi_{2}=\varepsilon L$, and $\phi_{2}-\phi_{s}=\phi_{f / s}$ we find that the potential drop across the metal/barrier layer $(\mathrm{m} / \mathrm{bl})$ interface to be:

$\phi_{m / f}=(1-\alpha) V-\beta p H-\varepsilon L-\phi_{f / s}^{0}$

where $\varepsilon$ is the electric field strength in the barrier oxide layer $(\mathrm{V} / \mathrm{cm})$, and $L$ is the barrier layer thickness (cm).

The parameter, $\varepsilon$, the electric field strength, is considered to be independent of distance through the barrier layer and to be independent of applied voltage, because of the occurrence of Esaki, tunneling $[13,14,33]$. Thus, this process is envisioned to buffer the electric field strength at some upper value that is 
below the dielectric strength at which dielectric breakdown occurs $(1<\varepsilon<5 \mathrm{MV} / \mathrm{cm})$, via field mediated, quantum-mechanical tunneling between the valence and conduction bands with the point defects acting as the inter-band gap states $[14,33]$, such that if any process or stress (e.g., applied potential) causes the field to increase the tunneling distance between the $\mathrm{CB}$ and $\mathrm{VB}$ decreases resulting in an exponential increase in the Esaki tunneling current and in a separation of charge that opposes the field.

The interfacial reactions that are responsible for the generation and annihilation of point defects and for the dissolution of the barrier layer, as envisioned in the PDM, are displayed in Figure 4. 
Meta

$\underline{\text { Barrier Layer }\left(\mathrm{MO}_{\chi / 2}\right)}$

- Solution

(1) $m+V_{M}^{\chi^{\prime}} \stackrel{k_{1}}{\longrightarrow} M_{M}+v_{M}+\chi e$

(2)

$$
m \stackrel{k_{2}}{\longrightarrow} M_{i}^{\chi+}+v_{M}+\chi e
$$

(3) $m \stackrel{k_{3}}{\longrightarrow} M_{M}+\frac{\chi}{2} V_{o}^{*}+\chi e$

(6) $V_{o}^{*}+H_{2} \mathrm{O} \stackrel{k_{6}}{\longrightarrow} \mathrm{O}_{O}+2 H^{+}$

(7) $\mathrm{MO}_{\chi / 2}+\chi H^{+} \stackrel{k_{7}}{\longrightarrow} M^{\delta+}+\frac{\chi}{2} H_{2} \mathrm{O}+(\delta-\chi) e^{\prime}$

$M_{M} \stackrel{k_{4}}{\longrightarrow} M^{\delta+}(a q)+V_{M}^{\chi^{\prime}}+(\delta-\chi) e^{\prime}$

$M_{i}^{\chi+} \stackrel{k_{5}}{\longrightarrow} M^{\delta+}(a q)+(\delta-\chi) e^{\prime}$

Movements of

Point Defects

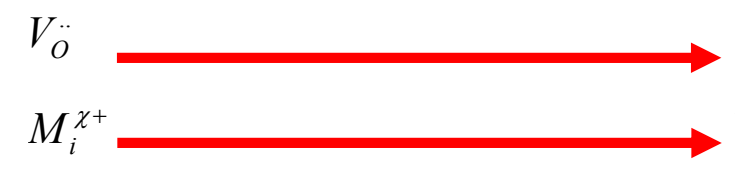

Migration flux n-type conductivity

Migration flux p-type conductivity<smiles>[AlH]</smiles>

Reactions 3 and 7 are lattice non- conservative; other reactions are lattice conservative

\begin{tabular}{|l|l|}
\hline$m:$ metal atom & $M^{\delta+}(a q):$ metal cation in solution \\
\hline$V_{M}^{\chi^{\prime}}:$ cation vacancy on the barrier layer & $O_{O}:$ oxygen anion on the barrier layer \\
\hline$M_{M}:$ metal cation on the barrier layer & $M_{i}^{\chi+}:$ interstitial cation \\
\hline$V_{M}^{\chi}:$ metal vacancy & $V_{O}:$ oxygen vacancy on the barrier layer \\
\hline
\end{tabular}

Figure 4: Reactions envisioned to occur at the metal/barrier layer $(\mathrm{m} / \mathrm{bl})$ and barrier layer/solution $(\mathrm{bl} / \mathrm{s})$ interfaces, according to the Point Defect Model, resulting in the generation and annihilation of the point defects. The migration fluxes are represented by red arrows for defects that account for $\mathrm{n}$ - or p-type conductivity in the barrier layer. 
As also noted above, the barrier layer of the passive film is either $p$ - or $n$-type in electronic character, depending upon the relative concentrations of cation vacancies (electron acceptors, p-type dopant) and oxygen vacancies and metal interstitials (electron donors, n-type dopants). Since the defects are generated or annihilated by the interfacial reactions depicted in Figure 4, the relative concentrations of the three defects in the barrier layer are sensitive functions of the kinetics of the reactions, as reflected in the values for the standard rate constants and the transfer coefficients. Furthermore, it is necessary to classify the interfacial reactions as to whether they are "lattice conservative (lc)" or "lattice nonconservative $(\ln c)$ "; a $l c$ reaction is one for which the boundary does not move upon the occurrence of the reaction, while a $l n c$ reaction results in boundary movement. Noting that the smallest structural increment of the barrier layer is one cation and $\chi / 2$ anions $\left(\mathrm{M}_{\mathrm{M}}+\chi / 2 \mathrm{O}_{\mathrm{O}}\right)$ or even $\left(\mathrm{V}_{\mathrm{M}}+\chi / 2 \mathrm{O}_{\mathrm{O}}\right)$ or $\left(\mathrm{M}_{\mathrm{M}}+\chi / 2 \mathrm{~V}_{\mathrm{O}}\right)$, but apparently not $\left(\mathrm{V}_{\mathrm{M}}+\chi / 2 \mathrm{~V}_{\mathrm{O}}\right)$, because vacancies can be considered to be real species, it is evident that Reactions (3) and (7) are non-conservative and account for the increase and the decrease of the layer thickness, respectively. The other reactions are all lattice conservative. It should be noted that the addition of Reactions (1) and (4), and Reactions (2) and (5), in Figure 2, so as to eliminate the defects, give the same overall Reaction (4), and the addition of Reactions (3) and (6) give Reaction (5), as previously noted $[13,14]$.

$m \longrightarrow M^{\delta+}(a q)+\delta e^{e}+v_{M}$

and

$m+\frac{\chi}{2} \mathrm{H}_{2} \mathrm{O} \longrightarrow\left[M_{M}+\frac{\chi}{2} \mathrm{O}_{O}\right]+\chi H^{+}+\chi e^{\prime}$

Thus, we conclude that the generation and annihilation of cation vacancies [Reactions (1) and (4)] and cation interstitials [Reactions (2) and (5)] are formally equivalent to the dissolution of the metal through a semi-permeable membrane in the form of the barrier layer. It is only Reactions (3) and (6), above, that result in the formation of the film, where $\left[M_{M}+\frac{\chi}{2} O_{O}\right]$ is formally equivalent to $\mathrm{MO}_{\chi / 2}$, as also noted above. Thus, in describing film growth/dissolution, we need to consider only two reactions, Reactions (3) and Reaction (7), Figure 4, regardless of which defect is most prevalent in the barrier layer (i.e. regardless of the electronic type).

The rate constants for the various reactions in the PDM presented above are functions of the applied potential, film thickness, and $p H$, depending upon the location of the reaction and have the form, see Table $3[13,14,21]$ :

$k_{\mathrm{i}}=k_{\mathrm{i}}^{0} \mathrm{e}^{a_{\mathrm{i}} V} \mathrm{e}^{b_{\mathrm{i}} L} \mathrm{e}^{c_{\mathrm{i}} P H}$ 
Thus, from Figure 4, the rate constants for Reactions (1), (2) and (3) depend on film thickness, as well as on the applied voltage and $p H$, because the potential drop across the $\mathrm{m} / \mathrm{bl}$ interface, which drives the reactions, depends upon the potential drop across the barrier layer and hence upon the film thickness as dictated by Equation (3) and summarized in Table 3. Other reactions, those at the bl/ol interface, depend upon the $\mathrm{pH}$ and applied potential, but not on the film thickness, because the relevant voltage at the point of application (the reference electrode in the solution, just outside the outer layer) has not been imposed across the barrier layer at the point of impact on the rate (the bl/s interface). Tables 3 and 4 summarize, for each reaction, the parameters involved in the rate constant expressions [13,14,21].

Table 3: Rate constant expressions: $k_{\mathrm{i}}=k_{\mathrm{i}}^{0} \mathrm{e}^{a_{i} V} \mathrm{e}^{b_{1} L} \mathrm{e}^{c_{p} p H} ; \alpha_{\mathrm{i}}$ : transfer coefficient; $\gamma=F / R T, R$ : perfect gas constant, $F$ : Faraday's constant, $T$ : temperature; $k_{\mathrm{i}}^{0}$ : standard rate constant, as described by the PDM $[24,25]$.

\begin{tabular}{|l|c|c|c|}
\hline \multicolumn{1}{|c|}{ Reaction } & $\boldsymbol{a}_{\mathbf{i}}\left(\mathbf{V}^{-\mathbf{1}}\right)$ & $\boldsymbol{b}_{\mathbf{i}}\left(\mathbf{c m}^{-1}\right)$ & $\boldsymbol{c}_{\mathbf{i}}$ \\
\hline (1) $m+V_{M}^{\chi^{\prime}} \stackrel{k_{1}}{\longrightarrow} M_{M}+v_{M}+\chi e^{\prime}$ & $\alpha_{1}(1-\alpha) \chi \gamma$ & $-\alpha_{1} \chi \varepsilon \gamma$ & $-\alpha_{1} \beta \chi \gamma$ \\
\hline (2) $m \stackrel{k_{2}}{\longrightarrow} M_{i}^{\chi^{+}}+v_{M}+\chi e^{\prime}$ & $\alpha_{2}(1-\alpha) \chi \gamma$ & $-\alpha_{2} \chi \varepsilon \gamma$ & $-\alpha_{2} \beta \chi \gamma$ \\
\hline (3) $m \stackrel{k_{3}}{\longrightarrow} M_{M}+\frac{\chi}{2} V_{o}+\chi e^{\prime}$ & $\alpha_{3}(1-\alpha) \chi \gamma$ & $-\alpha_{3} \chi \varepsilon \gamma$ & $-\alpha_{3} \beta \chi \gamma$ \\
\hline (4) $M_{M} \stackrel{k_{4}}{\longrightarrow} M^{\delta+}(a q)+V_{M}^{\chi^{\prime}}+(\delta-\chi) e^{\prime}$ & $\alpha_{4} \alpha \delta \gamma$ & & $\alpha_{4} \beta \delta \gamma$ \\
\hline (5) $M_{i}^{\chi+} \stackrel{k_{5}}{\longrightarrow} M^{\delta+}(a q)+(\delta-\chi) e^{\prime}$ & $\alpha_{5} \alpha \delta \gamma$ & & $\alpha_{5} \beta \delta \gamma$ \\
\hline (6) $V_{o ̈}+H_{2} O \stackrel{k_{6}}{\longrightarrow} O_{O}+2 H^{+}$ & $2 \alpha_{6} \alpha \Gamma \gamma$ & & $2 \alpha_{6} \beta \delta \gamma$ \\
\hline (7) $M O_{\chi / 2}+\chi H^{+} \stackrel{k_{7}}{\longrightarrow} M^{\delta+}+\frac{\chi}{2} H_{2} O+(\delta-\chi) e^{\prime}$ & $\alpha_{7} \alpha \delta \gamma$ & & $\alpha_{7} \beta \delta \gamma$ \\
\hline
\end{tabular}


Table 4: Standard rate constants for the interfacial defect generation/annihilation and film dissolution reactions as employed in the PDM. $k_{1}^{00}\left(\mathrm{~mol}_{\mathrm{cm}} \mathrm{cm}^{-2} \mathrm{~s}^{-1}\right)$ : base standard rate constant; $E_{a, \mathrm{j}}\left(\mathrm{kJ} \cdot \mathrm{mol}^{-1}\right)$ : Arrhenius activation energy for each reaction; $T_{0}$ : reference temperature.

\begin{tabular}{|c|c|}
\hline Reaction & $k_{\mathrm{i}}^{0}$ \\
\hline (1) $m+V_{M}^{\chi^{\prime}} \stackrel{k_{1}}{\longrightarrow} M_{M}+v_{M}+\chi e^{\prime}$ & $k_{1}^{00} \mathrm{e}^{-\frac{-\alpha_{1} \chi \gamma \varphi_{f / s}^{0}}{2}} \mathrm{e}^{\frac{-E_{a, 1}}{R T}\left(\frac{1}{T}-\frac{1}{T_{0}}\right)}$ \\
\hline (2) $m \stackrel{k_{2}}{\longrightarrow} M_{i}^{\chi+}+v_{M}+\chi e^{\prime}$ & $k_{2}^{00} \mathrm{e}^{-\alpha_{2} \chi \gamma \gamma \varphi_{f / s}^{0}} \mathrm{e}^{\frac{-E_{a, 2}}{R T}\left(\frac{1}{T}-\frac{1}{T_{0}}\right)}$ \\
\hline (3) $m \stackrel{k_{3}}{\longrightarrow} M_{M}+\frac{\chi}{2} V_{o}+\chi e^{\prime}$ & $k_{3}^{00} \mathrm{e}^{-\alpha_{3} \chi \gamma \varphi_{f / s}^{0}} \mathrm{e}^{\frac{-E_{a, 3}}{R T}\left(\frac{1}{T}-\frac{1}{T_{0}}\right)}$ \\
\hline (4) $M_{M} \stackrel{k_{4}}{\longrightarrow} M^{\delta+}(a q)+V_{M}^{\chi^{\prime}}+(\delta-\chi) e^{\prime}$ & $k_{4}^{00} \mathrm{e}^{\alpha_{4} \Gamma \gamma \phi_{f / s}^{0}} \mathrm{e}^{\frac{-E_{a, 4}}{R T}\left(\frac{1}{T}-\frac{1}{T_{0}}\right)}$ \\
\hline (5) $M_{i}^{\chi+} \stackrel{k_{5}}{\longrightarrow} M^{\delta+}(a q)+(\delta-\chi) e^{\prime}$ & $k_{5}^{00} \mathrm{e}^{\alpha_{5} \Gamma \gamma \phi_{f / s}^{0}} \mathrm{e}^{\frac{-E_{a, 5}}{R T}\left(\frac{1}{T}-\frac{1}{T_{0}}\right)}$ \\
\hline (6) $\mathrm{V}_{O}^{\prime \prime}+\mathrm{H}_{2} \mathrm{O} \stackrel{k_{6}}{\longrightarrow} \mathrm{O}_{O}+2 H^{+}$ & $k_{6}^{00} \mathrm{e}^{2 \alpha_{6} \gamma \gamma \varphi_{f / s}^{0}} \mathrm{e}^{\frac{-E_{a, 6}}{R T}\left(\frac{1}{T}-\frac{1}{T_{0}}\right)}$ \\
\hline (7) $M O_{\chi / 2}+\chi H^{+} \stackrel{k_{7}}{\longrightarrow} M^{\delta+}+\frac{\chi}{2} H_{2} O+(\delta-\chi) e^{\prime}$ & $k_{7}^{00} \mathrm{e}^{\alpha_{7}(\Gamma-\chi) \gamma \phi_{f / s}^{0}} \mathrm{e}^{\frac{-E_{a, 7}}{R T}\left(\frac{1}{T}-\frac{1}{T_{0}}\right.}$ \\
\hline
\end{tabular}

Amongst the many models that have been proposed to describe the passivity of metals, including the growth and breakdown of the passive film; only the Point Defect Model (PDM), developed by Macdonald et al [13-21], appears to account for all of the repeatedly-verified, experimental observations on the structure and properties of passive films [13,14]. The PDM describes the passive film in terms of fundamental chemical reactions and physico-electrochemical processes of the metal-passive film-solution interphase. The passive film has been found to form as a bi-layer, with the structure comprising an inner, defective metal oxide layer (primary passive film or barrier layer) that forms directly from the metal and a precipitated, outer layer that forms from the hydrolysis of cations ejected from the inner layer, as previously noted. The precipitated outer layer frequently incorporates species found in the bulk solution in contact with the metal or alloy that are not found in the alloy itself, while the barrier layer is found to contain only elements present in the metal substrate and not species present in the environment.

The PDM describes the growth and breakdown of passive films in terms of mass and charge fluxes via the transport of point defects (cation vacancies, cation interstitials, and oxygen vacancies) across the defect semiconducting barrier layer of the passive film, with the point defects (cation and oxygen vacancies and metal interstitials) being generated and annihilated by reactions at the barrier layer interfaces, Figure 1. From the defect generation and annihilation reactions at the barrier layer 
boundaries [metal/barrier layer $(\mathrm{m} / \mathrm{bl})$ interface and the barrier layer/outer layer (bl/ol) interface], equations describing mass and charge flux and reaction kinetics are derived, including equations for the rate of growth of the oxide into the metal at the $\mathrm{m} / \mathrm{bl}$ interface and the rate of dissolution of the barrier layer at the bl/ol interface. Further mathematical operations yield sets of equations for the steady-state passive current density and barrier layer thickness and for the response of the system to large and small, time-dependent, applied potential perturbations, as used in electrochemical studies, including chrono-amperometry [18], electrochemical impedance spectroscopy (EIS) [19] and cyclic voltammetry (CV) [20]. From these equations, and from the experimentally-measured current responses, including electrochemical impedance spectra, upon which the model is optimized, model parameters, such as standard rate constants, transfer coefficients, and interfacial potential drops may be determined.

In the particular case of the fretting corrosion of Type 316L SS in contact with PMMA, it is postulated that the rate of destruction of the barrier layer at the bl/ol interface is not only due to dissolution, but is also determined by wear, possibly involving oxide debris within the gap between the two surfaces, or is due to the direct interaction of the two surfaces themselves. In this particular case, as with most chromium-containing alloys, the compact inner layer (barrier layer) adjacent to the metallic surface is a thin chromic oxide (defective chromia, $\mathrm{Cr}_{2+\mathrm{x}} \mathrm{O}_{3-\mathrm{y}}$ ) $[19,34,35]$, in which the principal defect is the metal interstitial $(x>0)$, or oxygen vacancies $(y>0)$, or both $(x>0, y>0)$, with metal interstitials being commonly identified as the principal defect $[19,21]$. The barrier layers on a wide range of chromium-containing iron- and nickel-base alloys are invariably n-type in electronic character in a specific range of applied potentials [36-41], as expected from the defect assignment given above. As further noted above, the outer layer forms by the hydrolysis and precipitation of iron and nickel cations that are ejected from the barrier layer to form hydroxide, oxyhydroxide, or oxide corrosion products that also incorporate species from solution via co-precipitation. The outer layer is generally non-defective and commonly contains an mixed iron/nickel oxide phase [e.g., $(\mathrm{Fe}, \mathrm{Ni})_{2} \mathrm{O}_{3}$ ] with a different stoichiometry from that of the barrier layer. According to reports in the literature, for Type 316L SS, specifically, the barrier layer comprises chromium oxides, " $\mathrm{Cr}_{2} \mathrm{O}_{3}$ ", while the outer layer is composed of iron oxides, Fe (III) [37]. The results presented in this reference were obtained at high temperature (first vacuum annealed $1050{ }^{\circ} \mathrm{C}$ and exposition during 720 hours, at $p H=8$ and at temperature of $350{ }^{\circ} \mathrm{C}$ ), so that it is probable that the outer layer at ambient temperature and at $p H=8$ is iron hydroxide, $\mathrm{Fe}(\mathrm{OH})_{3}$, or iron oxyhydroxide, $\mathrm{FeOOH}$. While bulk chromic oxide is commonly ptype in electronic character, the defective chromic oxide barrier layer on chromium-containing alloys is invariably n-type, as determined by Mott-Schottky analysis [19,36-43], emphasizing that great care should be exercised when comparing the barrier layer of a passive with a bulk oxide, because the former is a "living entity" (it is being continually generated and destroyed), while the latter is "dead", and because the point defects are Schottky defects that are injected into the oxide by reactions occurring at the interfaces, rather than being Frenkel defects formed by the movement of an ion on the 
appropriate sublattice into an interstitial position. Furthermore, the interfacial defect generation/annihilation reactions occur in close juxtaposition (i.e., at interfaces that are separated by 1 $-3 \mathrm{~nm}$ ) (Figure 1), resulting in defect concentrations (typically $>10^{21} \mathrm{~cm}^{-3}$ ) that are orders of magnitude greater than those thermally induced in bulk oxides. A simple calculation shows that the concentration of cation sites on the cation sublattice of $\mathrm{Cr}_{2} \mathrm{O}_{3}$ is $4.14 \times 10^{22} \mathrm{~cm}^{-3}$. Thus, the defect concentration is estimated to be greater that $2.4 \%$ of the cation sites; far greater than is observed in bulk chromic oxide. Thus, bulk oxides have no comparable processes determining their defect structures. This electronic character is the direct result of the preponderance of metal interstitials and/or oxygen vacancies in the barrier layer. Thus, from $-0.5 \mathrm{~V}(\mathrm{SCE})$ to $0.2 \mathrm{~V}(\mathrm{SCE})$, which covers the passive range, the passive film on Type $316 \mathrm{~L}$ SS is n-type.

\subsection{Application of the Point Defect Model to the Prediction of Fretting Corrosion Degradation}

From Reactions (3) and (7), Figure 4, both of which are $l n c$, the thickness of the barrier layer of the passive film is expressed by Equation 7 [21].

$$
\frac{\mathrm{d} L}{\mathrm{~d} t}=\Omega k_{3}^{0} \mathrm{e}^{a_{3} V} \mathrm{e}^{b_{3} L_{s s}} \mathrm{e}^{c_{3} p H}-\Omega k_{7}^{0} \mathrm{e}^{a_{7} V} \mathrm{e}^{c_{7} p H}\left(\frac{C_{\mathrm{H}^{+}}}{C_{\mathrm{H}^{+}}^{0}}\right)^{n}-W=\frac{\mathrm{d} L^{+}}{\mathrm{d} t}-\frac{\mathrm{d} L^{-}}{\mathrm{d} t}
$$

Where the final term $(W)$ represents the wear rate, $V$ is the imposed voltage, $\Omega$ is the molar volume of the barrier layer per cation $\left(\mathrm{MO}_{\chi / 2}\right)$ and the other quantities are as defined above. The quantities, $\mathrm{d} L^{+} / \mathrm{d} t$ and $\mathrm{d} L^{-} / \mathrm{d} t$ represent the rates of film growth into the metal at the $\mathrm{m} / \mathrm{bl}$ interface and the rate of barrier layer destruction at the $\mathrm{bl} / \mathrm{s}$ interface, due to the combined effects of dissolution and wear. Here, it is noted that the outer layer is generally friable and is easily removed by the moving surfaces. Accordingly, it is assumed that the barrier layer alone is involved in fretting. The 'wear' term corresponds to the mechanical wear produced during fretting and has units of $\mathrm{cm} / \mathrm{s}$, but we emphasize that a synergistic relationship probably exists between mechanical wear and metal barrier layer dissolution. The values of $\mathrm{W}$ have been determined from the wear coefficient and wear depth data summarized in Table 2. The parameter, $n$, is the kinetic order of the passive layer dissolution reaction with respect to $H^{+}$. The medium conductivity is high, due to chloride, hydrogen, sodium, and calcium ions in the Ringer's solution. Indeed, the resistivity is equal to $35 \Omega . \mathrm{cm}$. Noting that the potential is measured outside of the crevice, the potential that is applied at the fretting surfaces must be corrected for the $I R$ potential drop down the crevice. Consequently, the actual potential applied in the wear zone, can be expressed as: 


$$
V=V^{\prime}-R A I
$$

where $R(\Omega)$ is the resistance of the solution between the wear zone and the reference electrode at location of the tip of the Luggin probe outside of the crevice between the stainless steel and PMMA surfaces, $A\left(\mathrm{~cm}^{2}\right)$ is the cross-sectional area of the crevice, $I\left(\mathrm{~A} . \mathrm{cm}^{-2}\right)$ is the corresponding current density, and $V^{\prime}$ is the applied voltage, (V(SHE)).

At steady-state, the thickness of the barrier layer is then given by the transcendental equation

$$
\Omega k_{3}^{0} \mathrm{e}^{a_{3}\left(V^{\prime}-R A I\right)} \mathrm{e}^{b_{3} L_{s s}} \mathrm{e}^{c_{3} p H}-\Omega k_{7}^{0} \mathrm{e}^{a_{7}\left(V^{\prime}-R A I\right)} \mathrm{e}^{c_{7} p H}\left(\frac{C_{\mathrm{H}^{+}}}{C_{\mathrm{H}^{+}}^{0}}\right)^{n}-W=0
$$

where the applied potential has been corrected for the $I R$ potential drop [44]. Equation (9) has no general analytical solution for $L_{s s}$, but solutions for limitingly high and low wear rates can be formulated. Thus, if $W$ is much greater than the dissolution term, Equation (7) reduces to

$$
\Omega k_{3}^{0} \mathrm{e}^{a_{3}\left(V^{\prime}-R A I\right)} \mathrm{e}^{b_{3} L_{s s}} \mathrm{e}^{c_{3} p H}=W
$$

or

$$
L_{s s}=-\frac{a_{3}}{b_{3}} V^{\prime}+\frac{a_{3}}{b_{3}} R A I-\frac{c_{3}}{b_{3}} p H+\frac{1}{b_{3}} \ln \left(\frac{W}{\Omega k_{3}^{0}}\right)
$$

Thus, if the wear rate, $W$, increases the barrier layer becomes thinner (note that $b_{3}$ is negative, Table 3 $[13,14])$ and the current increases [see Equation (14) below]. If $\mathrm{W}$ is sufficiently high, $L_{s s}$ could be equal to 0 and the barrier layer disappears; that is, the surface becomes depassivated [21]. On the other hand, if the wear rate is small compared to the dissolution rate, Equation (9) reduces to

$$
\Omega k_{3}^{0} \mathrm{e}^{a_{3}\left(V^{\prime}-R A I\right)} \mathrm{e}^{b_{3} L_{s s}} \mathrm{e}^{c_{3} p H}-\Omega k_{7}^{0} \mathrm{e}^{a_{7}\left(V^{\prime}-R A I\right)} \mathrm{e}^{c_{7} p H}\left(\frac{C_{\mathrm{H}^{+}}}{C_{\mathrm{H}^{+}}^{0}}\right)^{n}=0
$$

which yields

$$
L_{s s}=-\left(\frac{a_{3}-a_{7}}{b_{3}}\right) V^{\prime}+\left(\frac{a_{3}-a_{7}}{b_{3}}\right) R A I+\left(\frac{c_{7}-c_{3}}{b_{3}}\right) p H-\frac{1}{b_{3}} \ln \left(\frac{k_{3}^{0}}{k_{7}^{0}}\right)+\frac{1}{b_{3}} \ln \left(\frac{C_{\mathrm{H}^{+}}}{C_{\mathrm{H}^{+}}^{0}}\right)^{n}
$$

From Macdonald et al's. results [19,42], and from our preliminary work on optimizing the model on the experimental data, we have assembled a set of parameter values, as summarized in Table 5 , that will be used in the PDM. 
Table 5: Initial parameter values implemented in the PDM.

$$
\begin{array}{lllllll}
\alpha_{1} & \alpha_{2} & \alpha_{3} & \alpha_{4} & \alpha_{5} & \alpha_{6} & \alpha_{7} \text { (no unit) }
\end{array}
$$

$\begin{array}{lllllll}0.15 & 0.10 & 0.12 & 0.15 & 0.28 & 0.15 & 0.5\end{array}$

\begin{tabular}{lllllll}
$k_{1}^{00}$ & $k_{2}^{00}$ & $k_{3}^{00}$ & $k_{4}^{00}$ & $k_{5}^{00}$ & $k_{6}^{00}$ & $k_{7}^{00}\left(\mathrm{~mol} / \mathrm{cm}^{2} / s\right)$ \\
\hline
\end{tabular}

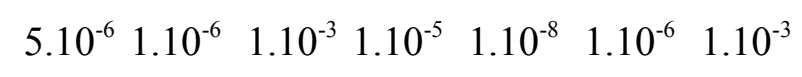

$\alpha$ (no unit) $\quad \beta(\mathrm{V}) \quad \varepsilon(\mathrm{V} / \mathrm{cm}) \quad \chi \quad \Gamma \quad T(\mathrm{~K}) \quad n$ (no unit) $\phi_{\mathrm{f} / \mathrm{s}}^{0}(\mathrm{~V})$

$\begin{array}{lllllllll}0.7 & -0.03 & 6.10^{6} & 2 & 3 & 298.15 & 0.6 & -0.1\end{array}$

The current density under any condition (steady-state or non steady-state) can be expressed by Equation (14) [44]:

$$
I=F\left[\begin{array}{l}
\chi k_{1}^{0} \mathrm{e}^{a_{1} V} \mathrm{e}^{b_{1} L_{s s}} \mathrm{e}^{c_{1} p H} C_{V_{M}}^{m / b l}+\chi k_{2}^{0} \mathrm{e}^{a_{2} V} \mathrm{e}^{b_{2} L_{s s}} \mathrm{e}^{c_{2} p H}+\chi k_{3}^{0} \mathrm{e}^{a_{3} V} \mathrm{e}^{b_{3} L_{s s}} \mathrm{e}^{c_{3} p H}+ \\
(\delta-\chi) k_{4}^{0} \mathrm{e}^{a_{4} V} \mathrm{e}^{c_{4} p H}+(\delta-\chi) k_{5}^{0} \mathrm{e}^{a_{5} V} \mathrm{e}^{c_{5} p H} C_{M_{i}}^{b l / o l}+ \\
(\delta-\chi) k_{7}^{0} \mathrm{e}^{a_{7} V} \mathrm{e}^{c_{7} p H}\left(\frac{C_{\mathrm{H}^{+}}}{C_{\mathrm{H}^{+}}^{0}}\right)^{n}
\end{array}\right]
$$

where one term exists for each of the reactions shown in Figure 4 that involves electrons. Noting that, at steady state, the Rates of Reactions (1), (2), and (3) must be equal to the rates of Reactions (4), (5), and (7), respectively, and after substituting Equation (8) the expression for the steady-state current takes the simpler form

$$
I_{s s}=\Gamma F\left[k_{4}^{0} \mathrm{e}^{a_{4}\left(V^{\prime}-R A I\right)} \mathrm{e}^{b_{4} L_{s s}} \mathrm{e}^{c_{4} p H}+k_{2}^{0} \mathrm{e}^{a_{2}\left(V^{\prime}-R A I\right)} \mathrm{e}^{b_{2} L_{s s}} \mathrm{e}^{c_{2} p H}+k_{3}^{0} \mathrm{e}^{a_{3}\left(V^{\prime}-R A I\right)} \mathrm{e}^{b_{3} L_{s s}} \mathrm{e}^{c_{3} p H}\right]
$$

where the parameters are as previously defined [14]. In this case, the first term on the right side corresponds to that for Reaction (4), Figure 4, whose rate is equal to that of Reaction (1) and the term for Reaction (7) has been replaced by that for Reaction (3). The rates of Reactions (3) and (7) must be equal in the steady-state, in order that the barrier layer has a constant thickness [see Equation (6)]. Note that it is assumed that wear does not result in the generation of electrons and hence Equation (15) does not contain a term that corresponds to wear. However, the steady state thickness of the barrier layer, $L_{s s}$, does depend upon the wear rate, $W$, Equation (11), and, because the thickness appears in the expression for the current [Equation (15)], so is the current, indirectly, a function of the wear rate, $W$, with the passive current density increasing with increasing wear rate.

As noted above, experimental studies have demonstrated that the principal defect in the defective $\mathrm{Cr}_{2+\mathrm{x}} \mathrm{O}_{3-\mathrm{y}}$ barrier layer is the metal interstitial $(x>y)[19,21,40]$ or possibly the oxygen 
vacancy. Accordingly, it may be assumed that the majority of the current is carried by the metal interstitials, as indicated in Ref. [21] for Alloy 22, and hence that the second term in Equation (15) is overwhelmingly greater than the other terms in the equation. Under these circumstances, Equation (15) reduces to an even more simple form, namely:

$$
I_{s s}=\Gamma F k_{2}^{0} \mathrm{e}^{a_{2}\left(V^{\prime}-R A I_{s s}\right)} \mathrm{e}^{b_{2} L_{s s}} \mathrm{e}^{c_{2} p H}
$$

For the case where $R=0$, substitution of Equation (13) into Equation (16) shows that the passive current density in the steady-state is constant and independent of potential, as has been previously shown $[13,14,21,44]$.

Equations (9) and (15) or (16) are a pair of simultaneous, transcendental equations that must be solved iteratively for $I_{s s}$ and $L_{s s}$. This is done by putting $I_{s s}=0$ on the right hand sides of Equations (9) and (15) and solving Equation (9) for $L_{s s}$ and then solving Equation (15) for $I_{s s}$. These are then substituted back into Equations (9) and (15) and the two equations are re-solved for $L_{s s}$ and $I_{s s}$. This process is continued, iteratively, until satisfactory convergence is obtained.

Because the majority of the current probably results from metal dissolution through the semipermeable barrier layer, it is possible through using Faraday's law to estimate the metal loss. Thus, the charge that is passed over time, $\tau$, is

$$
q=\int_{0}^{\tau} I_{s s} A \mathrm{~d} t
$$

from which the wear volume becomes

$$
\text { Wear Volume }=\frac{q \bar{M}}{F \rho \bar{\chi}}
$$

and the wear depth is

$$
\text { Wear Depth }=\frac{\text { Wear Volume }}{\text { A.w }}
$$

In these expressions, $\bar{M}$ is the composition -averaged atomic weight of the alloy, $\rho$ is the alloy density, $\bar{\chi}$ is the composition-averaged oxidation number for the alloy, $A$ is the fretting area, and $w$ is the width.

Finally, the wear zones were divided into 2 or 3 sub-zones. A gradient in solution resistance and $p H$, from the rim of the contact, Zone 1 , to the inner part of the wear track area, Zone 2, is assumed during modeling. These two zones are considered when the wear profile exhibits a valley. Three zones are employed when the wear profile exhibits a ' $W$ ' shape. The gradients of resistance and $p H$, and the wear rate, are postulated to account for this, particular wear shape. The evolution of the 
physical and chemical parameters is related to experimentally-observed processes, such as hydrogen evolution, as dictated by the $p H$ gradient from within to outside the contact zone. Figure 5 describes three zones, with Figure 5 a) depicting the juxtapositional relationship between zones and Figure 5 b) displaying an increment of surface, where the resistivity and the $p H$ are considered to be constant in modeling the wear with the PDM.

a)

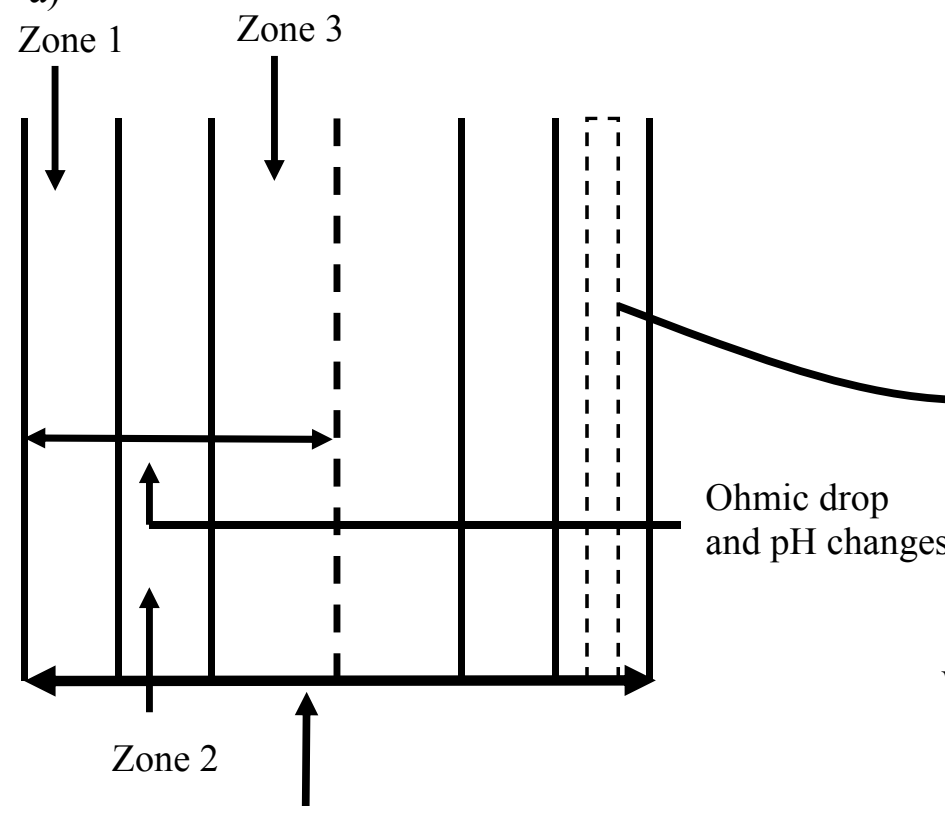

b)

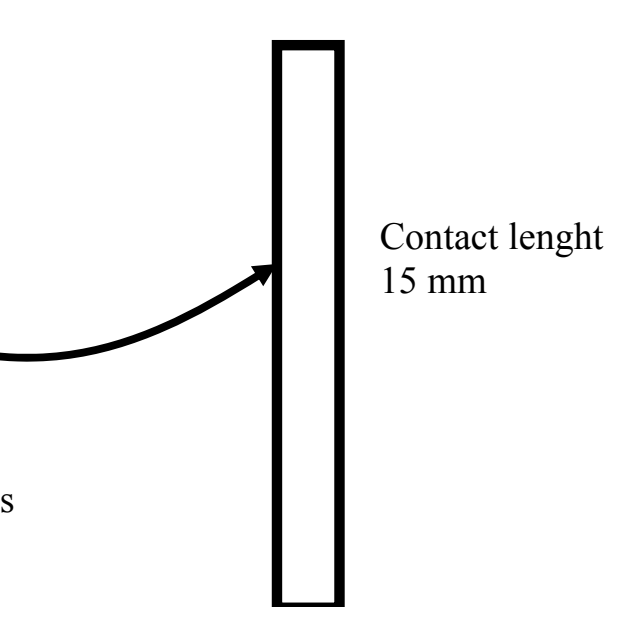

Width for wear calculation PDM: $1 \mu \mathrm{m}$

Fretting width

Figure 5: a) Contact zones, zone width subjected to fretting as determined by the displacement amplitude of the experiments, and the contact width between both materials; $b$ ) Increment of surface for calculating the current density and oxide layer thickness using the PDM.

From results taken from the literature and the hypotheses developed therefrom, a code was developed [47-49] to calculate the oxide layer thickness, the current density, and the wear depth profile, as described above.

\subsection{Oxide layer thickness and current density}

Figure 6 a) presents the oxide layer thickness as a function of $\mathrm{pH}$ and potential. Figure $6 \mathrm{~b}$ ) shows the current density calculated from the PDM. All results were obtained without considering wear, as predicted by the PDM using the parameter values summarized in Table 5. 
a)

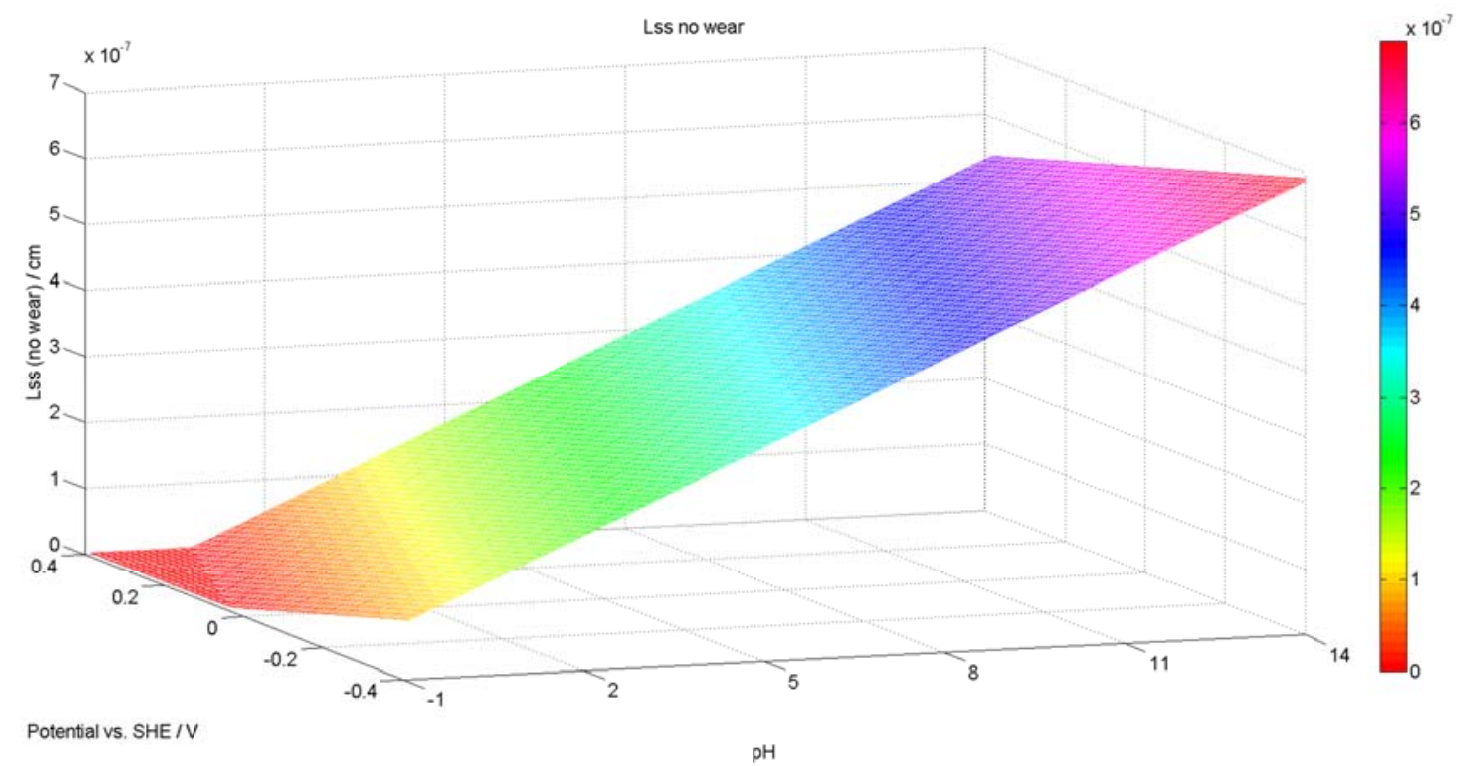

b)

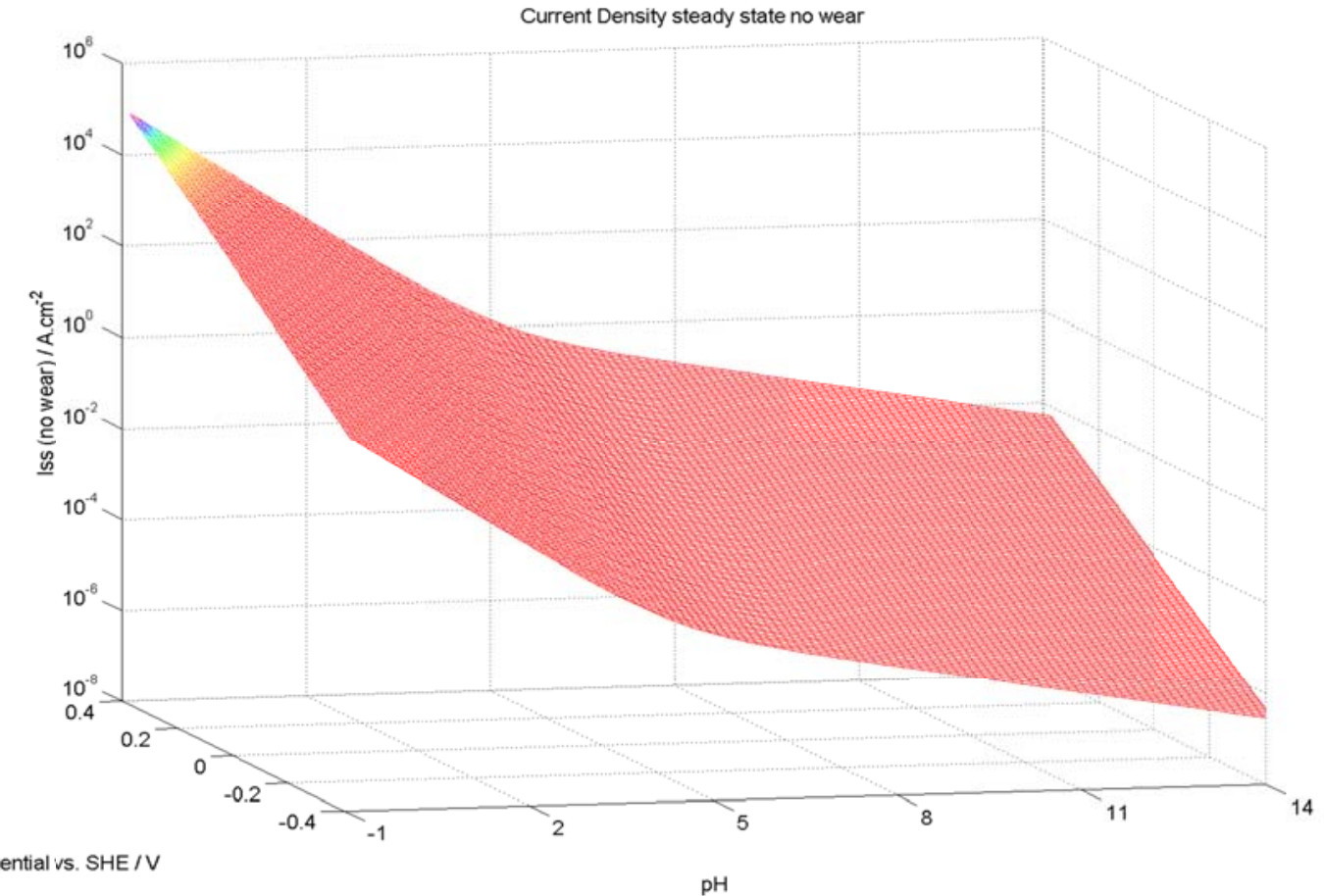

Figure 6: a) Oxide layer thickness, $L_{s s}$, as a function of $p H$ and applied potential $\mathrm{V}(\mathrm{SHE})$; b) Current density, $\log$ scale, as a function of $p H$ and applied potential V(SHE), without wear, for Type 316L SS. 
For the highest voltage and at a $\mathrm{pH}$ of $8.2, L_{s s}$ is comparable to that given by Marx et al. for iron (3 nm) [42], which is expected. Two physical parameters, especially fitted for better convergence of the PDM on the experimental data in the specific case of Type 316L SS considering dissolution alone (no wear), are: $\beta=-0.03$, and $\varepsilon=6 \times 10^{6} \mathrm{~V} \cdot \mathrm{cm}^{-1}$; all values are considered to be eminently reasonable. The results, Figure 6 a) show that, if the $\mathrm{pH}$ is lower than 1, the barrier layer does not exist, at any potential; that is, the surface is depassivated. In some cases, the oxide layer thickness is predicted to be negative, from which we conclude that the barrier layer of the passive film does not exist, the surface is depassivated, and the interfacial current is carried by metal dissolution alone; that is, active dissolution occurring without the presence of the protective oxide barrier layer. The negative layer thickness has no physical meaning, other than the barrier layer no longer exists, and hence $L_{s s}$ is set equal to zero. However, it should be noted that, under non-fretting conditions, an outer layer may exist on the surface that might provide some impediment to current flow. The current density is lower than $1 \mathrm{nA} . \mathrm{cm}^{-2}$, except for potentials higher than $0.3 \mathrm{~V}(\mathrm{SHE})$ and $p H$ values lower than 3 . At fixed $\mathrm{pH}$, $\mathrm{L}_{\mathrm{ss}}$ increases according to voltage, Figure 6a). The effect of ohmic drop should play a significant role on this evolution.

Figure 7 exhibits the steady state thickness and the current density as functions of $p H$ and applied potential V(SHE), taking into account the fitted wear $\left(\mathrm{cm} \cdot \mathrm{s}^{-1}\right)$. The $W$ factor, in this particular case, was chosen to describe the evolution of both $L_{s s}$ and the current density. The simulated data are of the same order of magnitude, but, nevertheless, are not exactly the same as, the experimental values. The zero value of $L_{s s}$ indicates that the growth of the barrier oxide layer is not promoted by fretting, i.e. wear. Indeed, the term $\frac{\mathrm{d} L^{-}}{\mathrm{d} t}$ is greater than $\left(\frac{\mathrm{d} L^{+}}{\mathrm{d} t}\right)_{L=0}$, which is the condition specified by the PDM for depassivation, and $L_{s s}$ is equal to zero. The same set of equations from the PDM did allow predicting the experimental values. Indeed, we did not measure the actual values of $\mathrm{pH}$ and resistance. Instead, we estimated the range and the evolution of these physical parameters and it is this approximation that is probably responsible for the simulated data not matching exactly the experimental wear data. However, one might expect that the $W$ term, alone, is probably not sufficient for describing all synergistic effects that could exist during the fretting corrosion process. For instance, the $W$ term is probably a function of the properties of the medium, i.e. $p H, V, T$ (temperature), medium composition and ionic strength [43]. This ionic strength is correlated to the chloride concentration in these typical experiments. It is found to have a huge impact on the corrosion resistance of the alloy, especially during wear. Thus, some another experiments will be detailed with different chloride concentrations in further investigations. A more exact treatment of fretting will incorporate synergism between the wear rate and dissolution via the properties of the solution, but that is beyond the current paper. 
These results show that the drastic dissolution is occurring at all applied potentials at the lowest $p H$, i.e. $p H<1$. This suggests that the $p H$ is lower than 1 in the contact zone, where the steel is subjected to friction. In the next paragraph, the actual wear profile, extracted from the profilometry analyses, will be compared with a simulated wear profile calculated from Equations (16), (17), (18) and (19).

a)

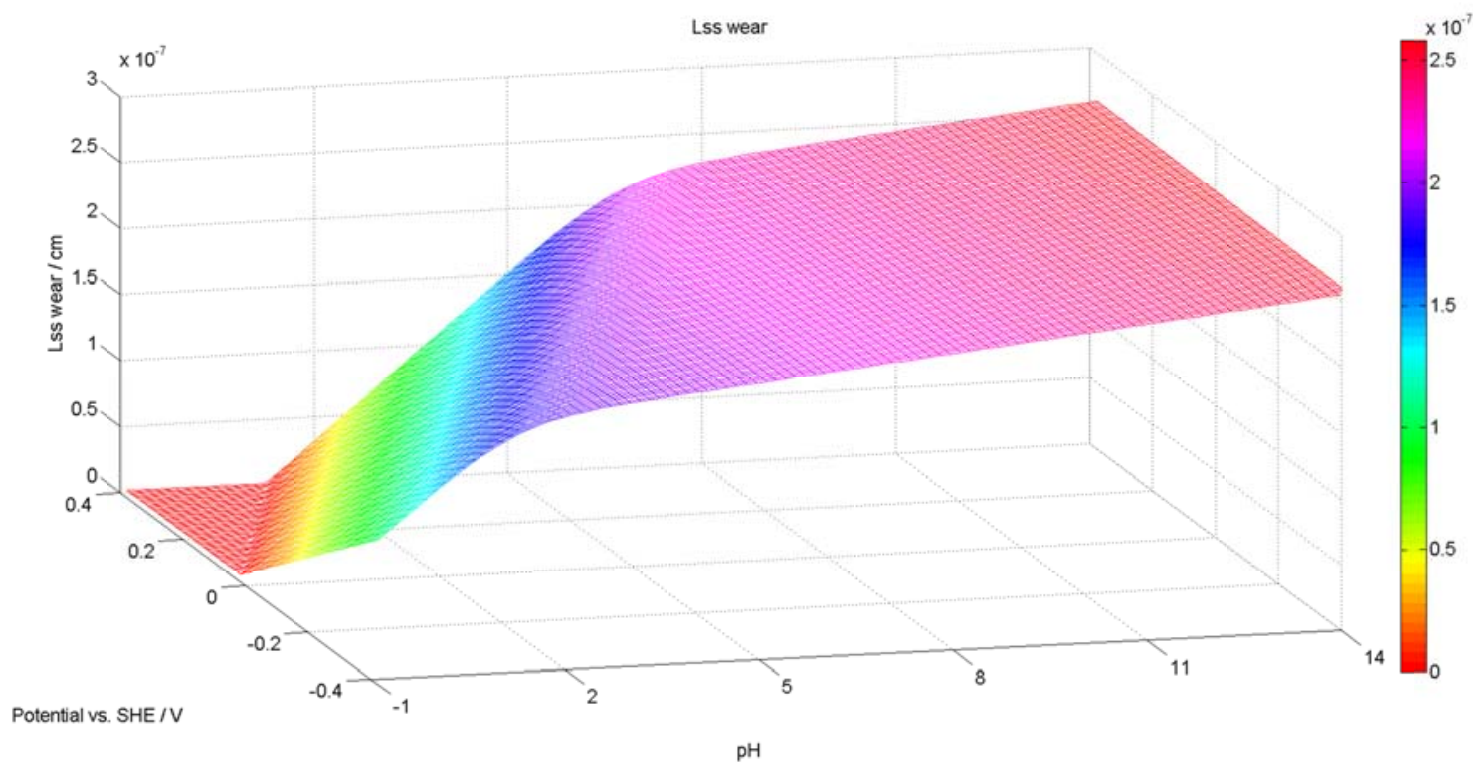

b)

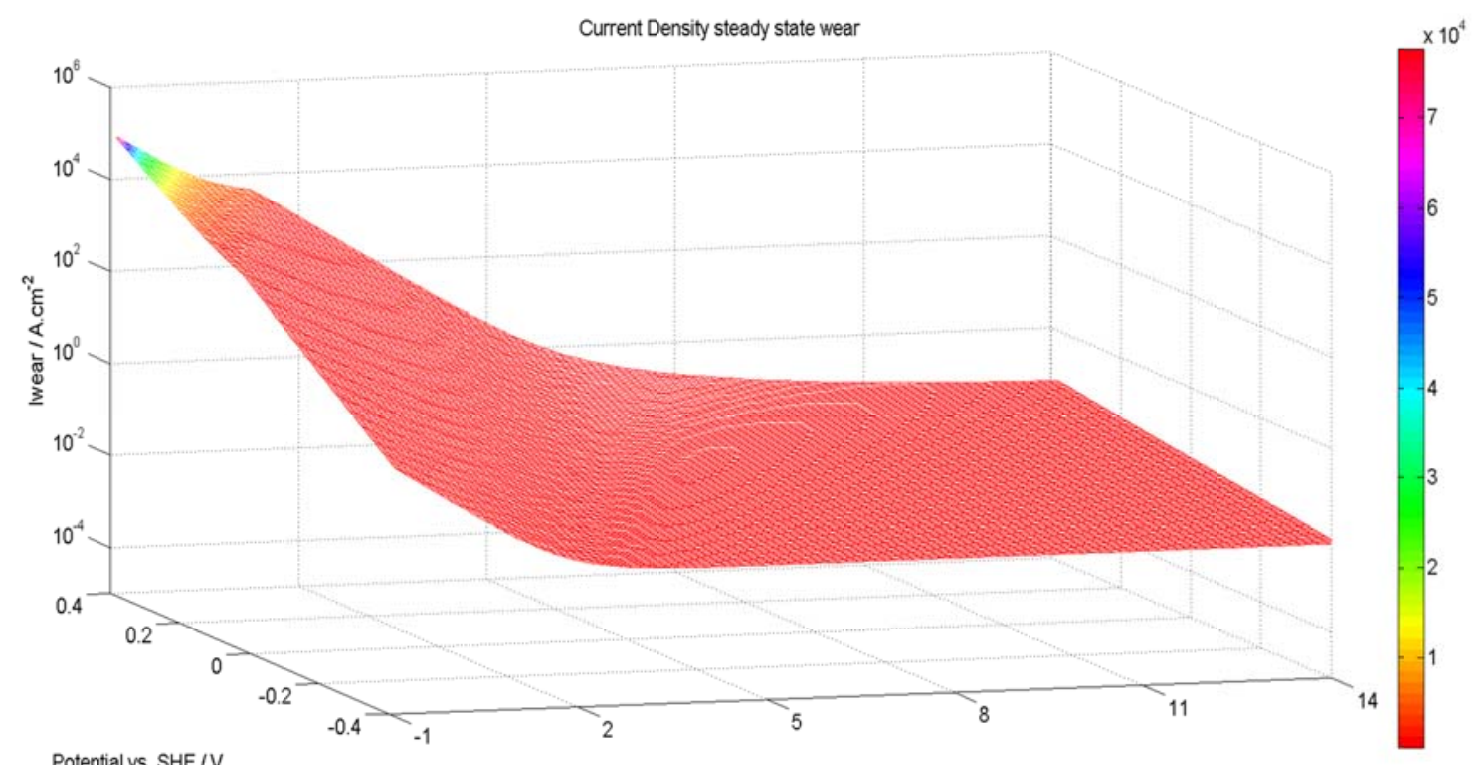

Potential vs. SHE/V 
Figure 7: a) Oxide layer thickness, $L_{s s}$, as a function of $p H$ and applied potential V(SHE) showing the impact of wear; b) Current density, log scale, as a function of $p H$ and applied potential V(SHE), with wear, for Type 316L SS.

In Figure $7 \mathrm{a}$ ), it is noted that oxide layer thickness at high potential and low $\mathrm{pH}$ is shown as being zero, and hence the barrier layer does not exist, because of the physical constraint that for passivity, $L_{s s}>0$. Fundamentally, this arises, because the destruction rate of the barrier layer at the barrier layer/solution interface under the influence of fretting, $\frac{\mathrm{d} L^{-}}{\mathrm{d} t}$, exceeds the rate of growth of the barrier layer into the steel substrate at the metal barrier layer interface and at zero barrier layer thickness, $\left(\frac{\mathrm{d} L^{+}}{\mathrm{d} t}\right)_{L=0} ;$ that is $\frac{\mathrm{d} L^{-}}{\mathrm{d} t}>\left(\frac{\mathrm{d} L^{+}}{\mathrm{d} t}\right)_{L=0}$. Increasing the wear rate, $W$, Equation (9), results in a shift of $L_{s s}$ towards less positive values (partial depassivation and a higher current density and, eventually, complete depassivation via loss of the barrier layer altogether) followed by a drastic decrease of $L_{s s}$ when the $\mathrm{pH}$ is close to 0 and the potential is higher than $0.2 \mathrm{~V}(\mathrm{SHE})$. The term "partial depassivation" describes the situation where the barrier layer, in the steady-state, has been thinned by fretting, compared with the absence of fretting, but where the barrier layer has not been completely destroyed. However the thinning of the barrier layer results in an exponential increase in the current density, as shown by Equation (15).

\subsection{Wear profile comparison at applied potentials equal to $0.25 \mathrm{~V}(\mathrm{SHE})$ and -0.15 V(SHE)}

The potential of $-0.15 \mathrm{~V}(\mathrm{SHE})$ corresponds to $\mathrm{n}$-type conductivity behavior of the passive film. It was postulated above that, in order to optimize the actual and experimental wear profiles, the solution resistance and $\mathrm{pH}$ must vary within the contact zone. These variations were assumed to be linear with distance from the contact rim to the inner part of the contact zone, all within the crevice formed by the stainless steel and the PMMA slide. In crevice corrosion phenomena, negative $p H$ values $\left(a_{H^{+}}>1\right)$ are often measured in the deepest zone, where the crevice effect occurs [50]. The hypothesis was formulated that the $p H$ varies from a value equal to 3 to a negative value, that is close to -1 , in the deepest zone. Moreover, the contribution of $p H$ to the current density is determined by the value of $\beta$. In this modeling work, the $\beta$ value was fitted to obtain the best convergence between the experimental and calculated damage (wear depth). For all further simulations and analyses, $\beta$ is taken to be $-0.03 \mathrm{~V}$, as listed in Table 5 , and $\varepsilon$ is taken to be equal to $6 \times 10^{6} \mathrm{~V} \cdot \mathrm{cm}^{-1}$. The value of $\beta$ is found to be typical of the values determined by the optimization of the PDM on electrochemical impedance spectroscopic data obtained on iron and stainless steels, for example, in contact with neutral and alkaline solutions, suggesting that $\beta$ is not strongly $p H$-dependent, as tacitly assumed in the PDM. 
Figure 8 presents a comparison between the actual and simulated wear depth profiles at an applied potential of $0.25 \mathrm{~V}(\mathrm{SHE})$. The actual wear profile exhibits a ' $\mathrm{W}$ ' profile. Because the damage is symmetric, comparison between theory and experiment has been carried out on only half of the entire worn zone, as indicated in Figure 8, because the wear track is considered as symmetric. The average depth measured from the non-worn surface, is different from the depth at the contact rim. We conclude that the contact rim is partially protected from corrosion, which may be due to an oxide deposit or by the fact that the $\mathrm{pH}$ and/or the potential are not sufficiently low to induce depassivation. This latter hypothesis is further confirmed by the image provided in Figure 1. Moreover, it is worth noting that the resistance must increase from the deepest zone of the ' $\mathrm{W}$ ' wear shape to the middle of the contact zone, in order to fit the model to the experimental data. This variation is physically realistic, because the metal dissolution should occur preferentially in the deepest zone having the greatest acidification, due to dissolution and metal cation hydrolysis to produce highly conductive protons. This increase in proton concentration decreases the solution resistance in the deepest zone, as noted above. Consequently, a positive gradient of resistance occurs from inside the contact to outside the wear zone.

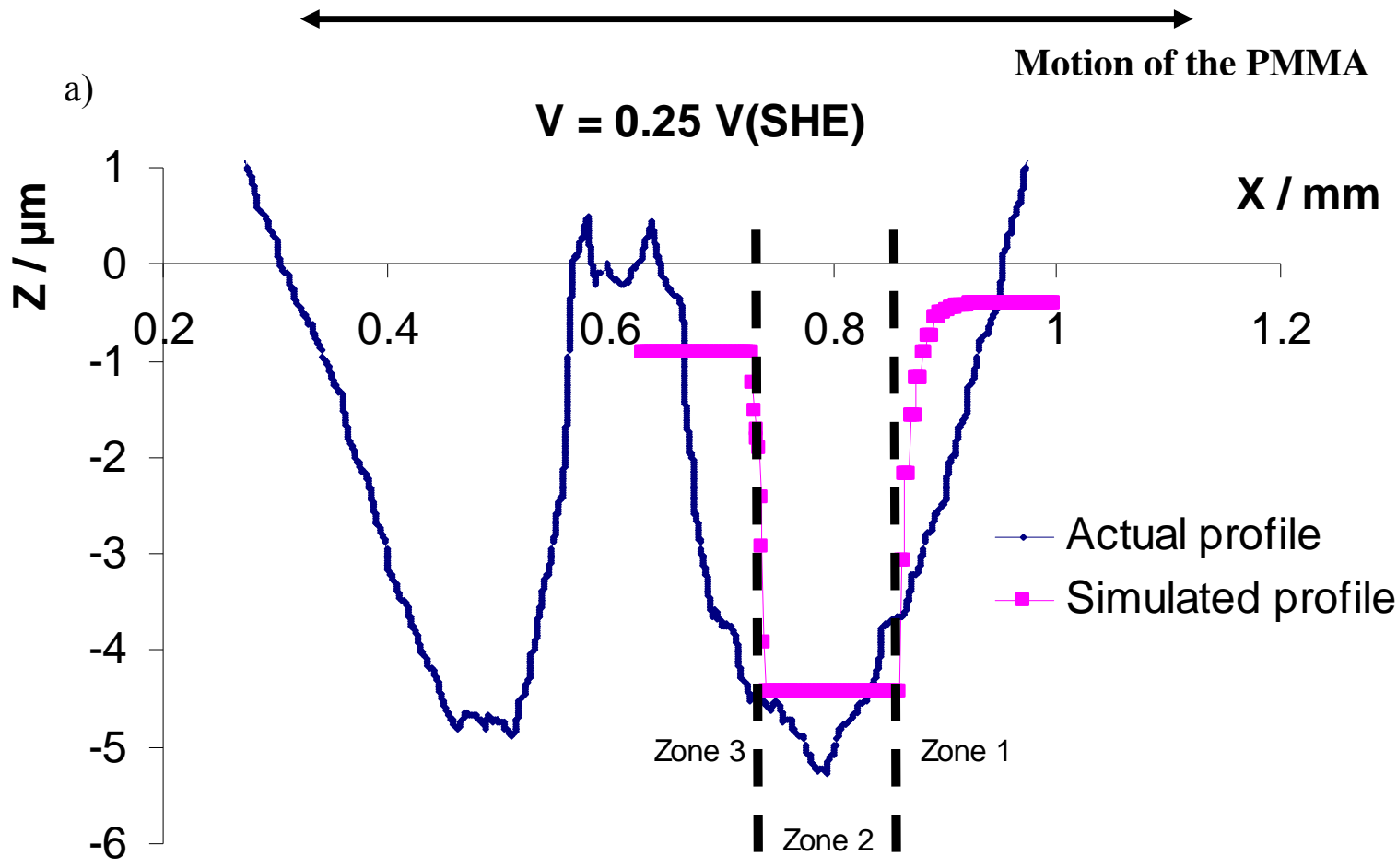

b)

\begin{tabular}{cccc} 
& Zone 3 & Zone 2 & Zone 1 \\
\hline pH & $(3 \leftarrow-1)$ & -1 & $(-1 \leftarrow 3)$ \\
Resist & $35 \leftarrow 0.5$ & 0.5 & $0.5 \leftarrow 35$
\end{tabular}

Figure 8: a) Actual (black) and simulated (pink) 2D profiles wear profiles (by the PDM); applied potential of $0.25 \mathrm{~V}(\mathrm{SHE})$, experimental duration of 20,000 seconds; b) $p H$ and resistance evolution according to different zones of fretting. 
Figure 9 exhibits simulated and actual wear depth profiles at $-0.15 \mathrm{~V}(\mathrm{SHE})$. The simulated profile is, approximately, in accordance with the actual profile. The $p H$ and resistance values allow prediction of the greatest depth of the wear track area. However, the comparison shows that, in Zone 1 , the fitted points, according to the PDM, are different from the actual points. This implies that the actual $\mathrm{pH}$ and/or resistance evolutions are not the same as those imposed.

\section{$V=-0.15 \mathrm{~V}(\mathrm{SHE})$}

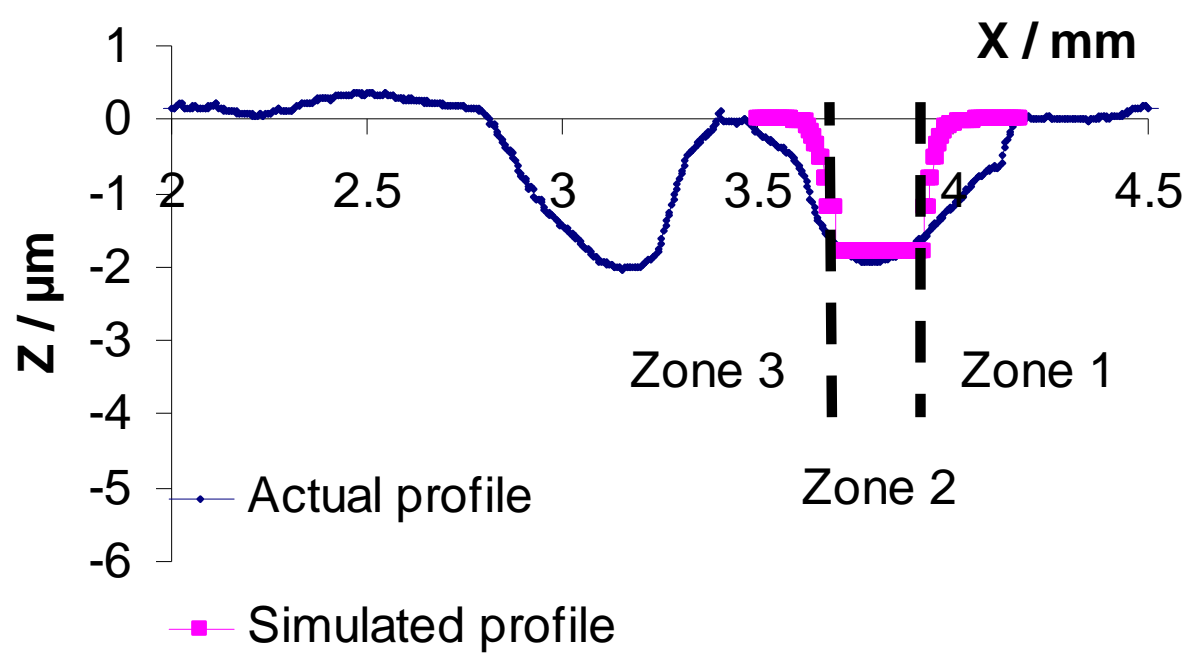

b)

\begin{tabular}{cccc} 
& Zone 3 & Zone 2 & Zone 1 \\
\hline pH & $(3 \leftarrow-0.5)$ & -0.5 & $(-0.5 \leftarrow 3)$ \\
Resist & $35 \leftarrow 0.5$ & 0.5 & $0.5 \leftarrow 35$
\end{tabular}

Figure 9: a) Actual (black) and simulated (pink) 2D profiles wear profiles (by the PDM); applied potential of $-0.15 \mathrm{~V}(\mathrm{SHE})$, experimental duration of 20,000 seconds of continuous fretting; b) $p H$ and resistance evolution for different zones of fretting.

To conclude, comparison between the experimental and simulated wear profiles is globally satisfactory. When the potential is equal to $0.25 \mathrm{~V}(\mathrm{SHE})$, the rim of the wear zone exhibits an agglomerate of debris. The model, in its present form, does not specifically account for the formation of this debris, which may be remnants of the barrier layer or oxides and oxyhydroxides that precipitate, because of the high metal cation concentrations, and, presumably will require the inclusion of more realistic mechanical concepts, in order for it to do so. At a potential of $-0.15 \mathrm{~V}$ (SHE), the 
wear, which results from synergism between corrosion and mechanics, is less than that which occurs at $0.25 \mathrm{~V}(\mathrm{SHE})$. The simulated profile is in better agreement with experiment. It is worth noting that the modeling reported here does not take into account debris transport and corrosion product deposition outside the contact zone. Additional work is required to study these phenomena, which are involved in friction and wear.

In the next section, the wear profile at $0.05 \mathrm{~V}(\mathrm{SHE})$ within the middle of the potential range from 0.25 to $-0.15 \mathrm{~V}$ (SHE), will be compared with a simulated profile. The conductivity type of the oxide layer is, again, n-type.

\subsection{Wear Profile Comparison at an Applied Potential Equal to 0.05 V(SHE)}

At this applied potential, i.e. $0.05 \mathrm{~V}(\mathrm{SHE})$, the profiles were compared for a fretting duration of 20,000 seconds. The $p H$ values are physically acceptable, even though they are negative $\left(a_{H^{+}}>1\right)$, Figure 10. At this potential, the wear volume was calculated from the optimized model and is compared with the actual wear volume.

\section{$V=0.05 \mathrm{~V}(\mathrm{SHE})$}

a)

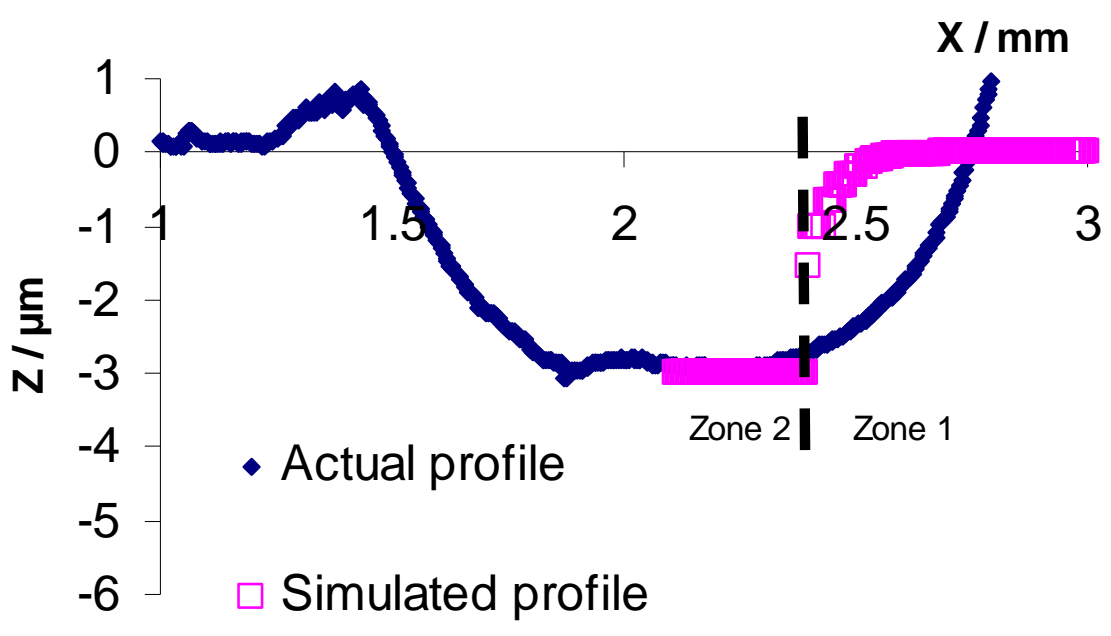

b)

\begin{tabular}{lcc} 
& Zone 2 & Zone 1 \\
\hline pH & -0.8 & $(-0.8 \leftarrow 3)$ \\
Resist & 0.5 & $0.5 \leftarrow 35$
\end{tabular}

Figure 10: a) Actual (black) and simulated (pink) 2D profiles wear profiles (by the PDM); applied potential of $0.05 \mathrm{~V}(\mathrm{SHE})$; experiments duration equal to 20,000 cycles of continuous fretting; $\mathrm{b}$ ) $p H$ and resistance evolution for different zones of fretting. 
Furthermore, Figure 11 presents a comparison between the simulated and the actual wear volumes. The difference, between both values of wear volume, is relatively small for this type of analysis, lower than $30 \%$, for potentials equal to -0.15 and $-0.05 \mathrm{~V}$ (SHE). These results highlight that the corrosion process is quite well-described at both potentials. However, for a potential of 0.25 $\mathrm{V}(\mathrm{SHE})$, the simulated wear volume does not agree well with the experimental value. The n-type conductivity of the oxide film should be discussed, at this point.

The n-type conductivity domain exists until $0.2 \mathrm{~V}(\mathrm{SCE})$, at which point cation vacancies become the dominant point defect in the barrier layer, because of the potential-mediated ejection of chromium from the barrier layer as the $\mathrm{Cr}(\mathrm{VI})$ species, $\mathrm{CrO}_{4}{ }^{2-}$. This process has been previously identified [21] as being responsible for the destruction of the barrier layer and the onset of transpassive dissolution, but it has not yet been incorporated into the current model. Thus, $0.25 \mathrm{~V}(\mathrm{SCE})$ can be considered to be the boundary between n-type and p-type conductivity and also as the upper limit of passivity. This phenomenon is readily incorporated into the model by inserting $\delta=6$ and $\chi=3$, corresponding to oxidative dissolution of the $\mathrm{Cr}(\mathrm{III})$ barrier layer $\left(\mathrm{Cr}_{2} \mathrm{O}_{3}\right)$ to form the $\mathrm{Cr}(\mathrm{VI})$ oxyanions, $\mathrm{CrO}_{4}{ }^{2-}$ in the solution, into parameter $a_{7}$ in Equation (9). Consequently, the dissolution term becomes large and the condition for oxidative depassivation (transpassive dissolution [21]) is achieved. This issue, as well as any synergism between dissolution and wear, will be explored in a future paper, but it is worth noting that inclusion of transpassive dissolution will greatly increase the calculated wear volume and possibly bring the simulated point into much better agreement with that measured, as shown in Figure 11.

As depicted in Figure 1, the wear track area, at this applied potential, exhibits great metal loss, which is consistent with depassivation and transpassive dissolution [21], as noted above. Above 0.1 $\mathrm{V}(\mathrm{SHE})$, the wear is drastically accelerated by mechanical friction, due to a decrease in the thickness of the barrier layer for potentials below the critical potential for transpassive dissolution, leading to complete destruction of the barrier layer at $E=E_{\text {trans }}$ [see Equation (9)]. As corrosion is promoted at this applied potential, the degradation of the metal is due principally to transpassive dissolution and not to wear. 


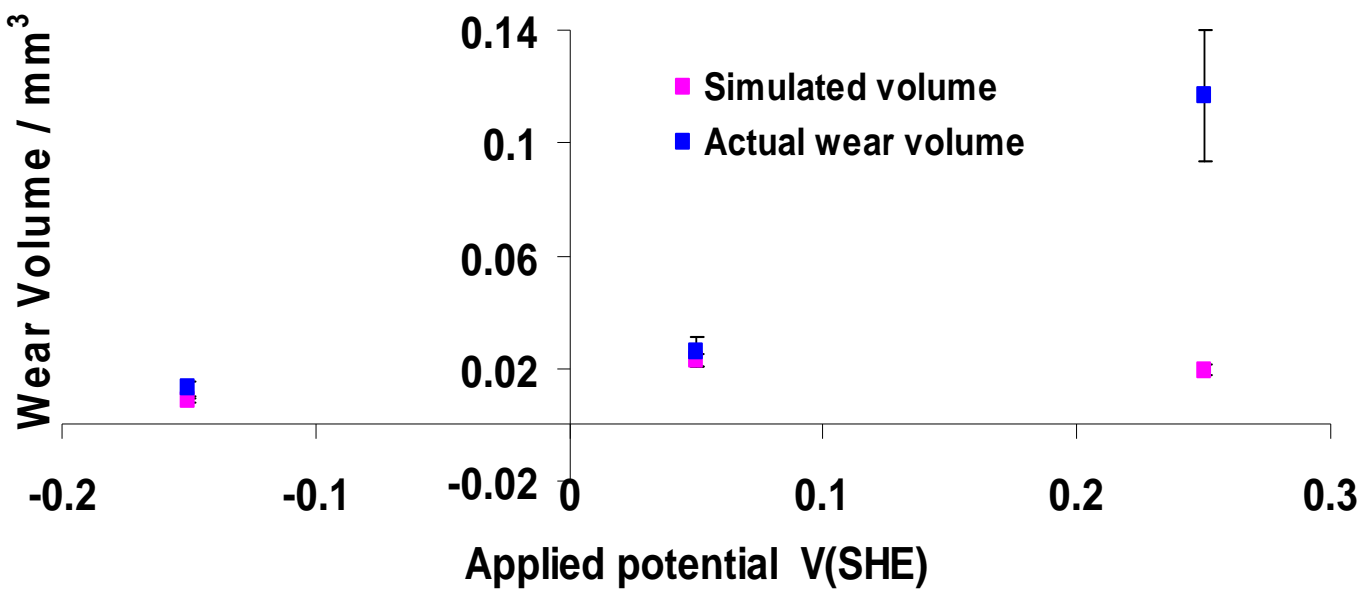

Figure 11: Experimental and simulated wear volumes as a function of applied potential after 20,000 cycles of continuous fretting.

Figure 12 presents a summary of the principal phenomena that are postulated to occur in the wear zone during fretting corrosion of Type 316 SS on PPMA in Ringer's solution. Two findings of this work must be highlighted: the $p H$ and solution resistance seem to decrease with distance into the wear zone (crevice), as noted above, and depassivation appears to be the principal damaging mechanism. With respect to the $p H$, it has been possible to obtain agreement between the actual and simulated depth of wear, only if the $p H$ is assumed to have a value as low as $-1 . p H$ values of this magnitude have been reported in classical crevice corrosion studies [50,51].

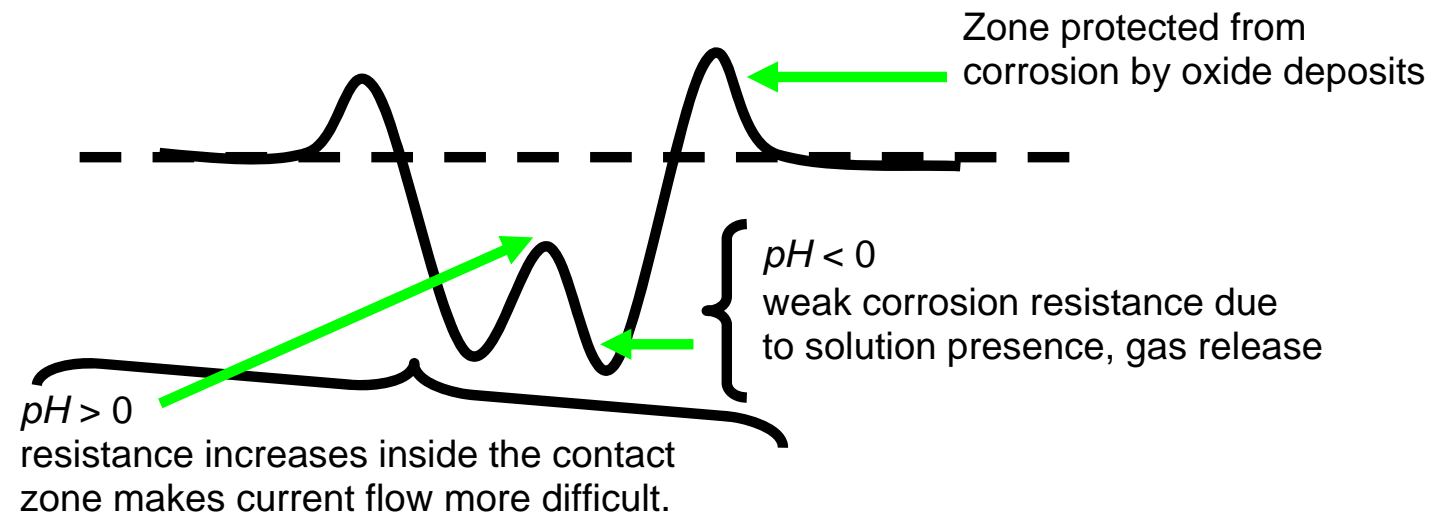

Figure 12: Schematic illustration of the different phenomena that are postulated to be involved in the formation of the wear zone by fretting in Ringer solution. 
Table 6 summarizes the different reactions that occur in a solution containing a high chloride concentration. These reactions are consistent with an acidification of the medium [50]. It is worth noting that these reactions have not been considered in this study. Only the $p H$ was specified, not the mechanism by which it is lowered. Accordingly, uninhibited metal dissolution may occur to generate a high local concentration of $H^{+}$via hydrolysis of $\mathrm{Cr}^{3+}$, thereby leading to the observed, low $p H$. The debris that is observed just outside of the rim most likely originates from the hydrolysis of $M^{z^{+}}$and the subsequent precipitation of hydroxides, oxyhydroxides, and oxides as the $\mathrm{Cr}^{3+}$ ions are transported from the crevice into the higher $\mathrm{pH}$ medium that exists outside of the crevice. These factors and mechanisms will be incorporated into future modeling work in this program. 
Table 6: Medium acidification resulting from metal dissolution in a solution containing high chloride concentration.

$$
\begin{aligned}
& \mathrm{nH}_{2} \mathrm{O}+\mathrm{M}^{\mathrm{z}+} \rightarrow\left[\mathrm{M}\left(\mathrm{H}_{2} \mathrm{O}\right)_{\mathrm{n}}\right]^{\mathrm{z}+} \\
& {\left[\mathrm{M}\left(\mathrm{H}_{2} \mathrm{O}\right)_{\mathrm{n}}\right]^{\mathrm{z+}}+\mathrm{hH}_{2} \mathrm{O} \rightarrow\left[\mathrm{M}\left(\mathrm{H}_{2} \mathrm{O}\right)_{\mathrm{n}-\mathrm{h}}(\mathrm{OH})_{\mathrm{h}}\right]^{(\mathrm{z}-\mathrm{h})+}+\mathrm{hH}_{3} \mathrm{O}^{+}} \\
& {\left[\mathrm{M}\left(\mathrm{H}_{2} \mathrm{O}\right)_{\mathrm{n}}\right]^{\mathrm{z+}}+\mathrm{mCl}^{-} \rightarrow\left[\mathrm{M}\left(\mathrm{H}_{2} \mathrm{O}\right)_{\mathrm{n}-\mathrm{m}}(\mathrm{Cl})_{\mathrm{m}}\right]^{(\mathrm{z}-\mathrm{m})^{+}}+\mathrm{mH}_{2} \mathrm{O}} \\
& {\left[\mathrm{M}\left(\mathrm{H}_{2} \mathrm{O}\right)_{\mathrm{n}-\mathrm{m}}(\mathrm{Cl})_{\mathrm{m}}\right]^{(\mathrm{z}-\mathrm{m})^{+}}+\mathrm{hH}_{2} \mathrm{O} \rightarrow\left[\mathrm{M}\left(\mathrm{H}_{2} \mathrm{O}\right)_{\mathrm{n}-\mathrm{m}-\mathrm{h}}(\mathrm{Cl})_{\mathrm{m}}(\mathrm{OH})_{\mathrm{h}}\right]^{(\mathrm{z}-\mathrm{m}-\mathrm{h})^{+}}+\mathrm{hH}_{3} \mathrm{O}^{+} .}
\end{aligned}
$$

The evolution of the solution resistance within the wear track area can be attributed to two factors, noting that the wear shape is ' $\mathrm{W}$ ' in form. Thus, while the solution can access the deepest zones of the ' $\mathrm{W}$ '-shaped crevice, solution transport is more difficult at the contact centre, because the materials are in very close proximity. Moreover, the acidification that occurs in the deepest zones results in an increase in the conductivity, thereby decreasing the solution resistance. As the centre zone is less deep, the depression of the $p H$ presumably is less severe. In order to assess the veracity of this conclusion, it might be possible to employ a hydrogenated palladium wire [50], oxidized iridium [51], or oxidized tungsten [52] for $p H$ measurements within the wear zone, provided that an accurate correction for the $I R$ potential drop down the crevice to the tip of the Luggin probe of the reference electrode can be made. Indeed, the potential of these pH sensors vs. that of a reference electrode, vary in a Nernstian fashion with the proton activity, and hence with $p H$, with the activity of hydrogen in the metal lattice, in the case of the Pd-H sensor, being established and controlled by the reaction xPd + $\mathrm{H}=\mathrm{Pd}_{\mathrm{x}} \mathrm{H}$, within the $\alpha+\beta$ region of the $\mathrm{Pd}-\mathrm{H}$ phase diagram [50]. These experiments are currently being planned.

This first attempt at the use of the PDM to model fretting corrosion wear, involved 7 reactions at the metal/barrier layer and barrier layer/solution interfaces. One might expect that chloride ion may participate in these reactions by, for example, being inserted in the reaction mechanisms. Indeed, Reaction 6, $\quad \mathrm{V}_{\mathrm{O}}^{*}+\mathrm{H}_{2} \mathrm{O} \stackrel{\mathrm{k}_{6}}{\longrightarrow} \mathrm{O}_{\mathrm{O}}+2 \mathrm{H}^{+}$, could be modified to read $\mathrm{V}_{\mathrm{O}}^{*}+\mathrm{Cl}^{-} \bullet \mathrm{nH}_{2} \mathrm{O} \stackrel{\mathrm{k}_{6}}{\longrightarrow} \mathrm{Cl}_{\mathrm{O}}^{\bullet}+\mathrm{nH}_{2} \mathrm{O}$, as has been done in the theory of passivity breakdown $[13,14]$. While this latter reaction is not involved in the current density expression, anion adsorption into surface oxygen vacancies leads to the continuous generation of cation vacancies at the bl/ol interface. These cation vacancies are transported by migration across the barrier layer to the $\mathrm{m} / \mathrm{bl}$ interface. Ordinarily, the cation vacancies are annihilated by Reaction 1, Figure 4. However, if the flux of vacancies is sufficiently large, Reaction 1 may not be able to annihilate all of the vacancies arriving at the interface, with the excess vacancies condensing on the cation sublattice at the interface to form a 
local void. This void causes the barrier layer to separate from the substrate metal and hence prevents the film from growing into the metal via the occurrence of Reaction 3, Figure 4. Because the barrier layer continues to dissolve at the barrier layer-solution interface, the "cap" over the vacancy condensate thins and eventually ruptures to mark a passivity breakdown event. This is the passivity breakdown mechanism, as proposed by the Point Defect Model [13,14], does result in a sharp enhancement of the current density. Thus, in the low $\mathrm{pH}$, low potential, and high $\left[\mathrm{Cl}^{-}\right]$environment that develops in the wear track, the barrier layer will be subjected to chloride-induced passivity breakdown [13,14], which along with acid depassivation, and transpassive dissolution at high potentials [21], will insure that the metal exists in the active state.

The value of the PDM is that it links atomic phenomena, such as vacancies formation with macroscopic phenomena, such as dissolution, in order to account for corrosion and wear observed under fretting corrosion conditions. This first attempt to apply the PDM to account for fretting corrosion has led to an assessment of the various assumptions, upon which the model is based and to a comparison of the model predictions with experimental results. With respect to the present work, the most important prediction is that the Type 316L SS will be depassivated in the wear track and that this phenomenon results in the observed geometry of attack. Indeed, a gas is evolved during fretting corrosion, which is most likely hydrogen, demonstrating that the metal surfaces in the wear track are in the active, not passive, state, as predicted by the PDM in this paper.

\section{4- Conclusions}

This study was focused on using the PDM to interpret and predict fretting corrosion (wear) occurring on Type 316L stainless steel by frictional contact with PMMA under cyclical displacement loading conditions. Optimization of the PDM on the measured wear profiles has yielded all of the PDM parameters that are required to describe the evolution of fretting corrosion damage.

In the case of an n-conductivity type barrier oxide layer of the passive film, modeling the total wear due to corrosion and mechanical abrasion has produced two important results. First of all, the $p H$ was found to decrease from the contact rim to the deepest zone within the ' $\mathrm{W}$ ' wear-shaped crevice. The current flow is more difficult at the contact center, because of the close proximity of the surfaces, due to the highest contact pressure at the center. Thus, at first sight, it might be expected that corrosion would be less intense and consequently the $\mathrm{pH}$ might be higher, because of the lower concentration of cations undergoing hydrolysis. On the other hand, one might also expect that the $p H$ should decrease into the deepest zone, and that the decrease could be correlated with crevice corrosion that should occur in this zone, even with the close juxtapositions of the steel and PPMA surfaces. The experimental evidence for a lower $p H$ is the release of gaseous hydrogen, indicating that the creviced wear zone had become very acidic and the steel surface has been depassivated to corrode in the active state. The second piece of evidence comes from the PDM. In order to fit the model, the $p H$ is 
required to reach negative values, again indicating significant acidification. Furthermore, the resistance follows the same gradient. The variations of both physical properties (resistance and $p H$ ) had to be optimized, in order to achieve good agreement between the simulated and the actual 2D wear profiles. This conclusion would be greatly supported if, for example, local measurements of $p H$ were available. These experiments are currently being planned.

\section{Acknowledgements}

The authors are grateful to Research Department, Region Rhône-Alpes, France, for granting a stay at the Pennsylvania State University, PA, USA. D. D. Macdonald also gratefully acknowledges his appointment as Adjunct Chair Professor in the Center for Research Excellence in Corrosion in the Research Institute, King Fahd University of Petroleum and Minerals, Dhahran, Saudi Arabia starting in December 2010.

\section{References}

1. M.M. Stack, N. Corlett, S. Zhou, Wear 203-204 (1997) 474

2. M.M. Stack, S.M. Abdelrahman, B.D. Jana, Wear 268 (2010) 533

3. M.M. Stack, G.H. Abdulrahman, Tribology International 43 (2010) 1268

4. L. Pidhorz, in: Conférences d'enseignement 1993, Cahiers d'enseignement de la SOFCOT $\mathrm{n}^{\circ} 44$, Expansion Scientifique Française, Paris, 1993, 31.

5. F. Langlais, in: Conférences d'enseignement 1983, Cahiers d'enseignement de la SOFCOT $\mathrm{n}^{\circ}$ 19, Expansion Scientifique Française, Paris, 1983, 15.

6. R.B. Waterhouse, Wear 100 (1984) 107

7. R.B. Waterhouse, Int. Mater. Rev. 37 (1992) 77

8. R.B Waterhouse, Fretting Corrosion, Pergamon Press, Oxford, 1975, 4

9. J.S. Kawalec, S.A. Brown, J.H. Payer, K. Merritt, J. Biomed. Mater. Res. 11 (1995) 867

10. C.N. Kraft, B. Burian, O. Diedrich, M.A. Wimmer, J. Mater. Sci. - Mater. Med. 12 (2001) 1057

11. C.H. Lohmann, D.D. Dean, G. Köster, D. Casasola, G.H. Buchhorn, U. Fink, Z. Schwartz, B.D. Boyan, Biomaterials 23 (2001) 1855

12. D. Kaczorowski, J.Ph. Vernot, Tribology International 39 (2006) 1286

13. D.D. Macdonald, J. Electrochem. Soc. 139 (1992) 3434

14. D.D. Macdonald, Pure Appl. Chem. 71 (1999) 951-978

15. C.Y. Chao, L.F. Lin, D.D. Macdonald, J. Electrochem. Soc. 128 (1981) 1187

16. L.F. Lin, C.Y. Chao, D.D. Macdonald, J. Electrochem. Soc. 128 (1981) 1194

17. C.Y. Chao, L.F. Lin, D.D. Macdonald, J. Electrochem. Soc. 129 (1982) 1874

18. D.D. Macdonald, M. Al-Rafaie, G.R. Engelhardt, J. Electrochem. Soc. 148 (9) (2001) B343

19. D.D. Macdonald, A. Sun, N. Priyantha, P. Jayaweera, J. Electroanal Chem. 572 (2) (2004) 421 
20. J. Bao, D.D. Macdonald, J. Electrochem. Soc. (2010) in review

21. D. D. Macdonald, J. Electrochem. Soc. 153 (7) (2006) B213

22. J. Geringer, B. Forest, P. Combrade, Wear 259 (2005) 943

23. M. Pourbaix, Gauthier-Villars, Paris, 1963.

24. J-P. Jolivet, De la solution à l'oxyde Condensation des cations en solution aqueuse-Chimie de surface des oxydes, CNRS Eds, Paris, 1994, 68-111

25. J. Geringer, Fretting corrosion of biomaterials, $\mathrm{PhD}$ thesis, $\mathrm{N}^{\circ}$ order: 391SGM; http://tel.archives-ouvertes.fr/docs/00/48/94/71/PDF/J-Geringer-051214.pdf

26. J. Geringer, B. Forest, P. Combrade, Wear 261 (2006) 971

27. H.W. Pickering, Corros. Sci. 29 (1989) 325

28. H.W. Pickering, K. Cho, E. Nystrom, Corros. Sci. 35 (1993) 775

29. B. G. Ateya, H. W. Pickering, Corros. Sci. 37 (1995) 1443

30. H.W. Pickering, Mater. Sci. Eng., A, 198 (1995) 213

31. M.I. Abdulsalam, H.W. Pickering, Corros. Sci. 41 (1998) 351

32. A.M. Al-Zahrani, H.W. Pickering, Electrochim. Acta 50 (2005) 3420

33. S. M. Sze and K. K. Ng, Physics of Semiconductor Devices, Wiley, $3^{\text {rd }}$ Edit.,New York, 2006.

34. C.R. Clayton, L. Rosenzweig, M. Oversluizen Y.C. Lu, Inhibition and Passivation, Electrochemical Society, Eds McCafferty and R.J. Brodd, Pennington NJ, 1986

35. I. Olejford and L. Wegrelius, Corros. Sci. 38 (1996) 1203

36. M.F. Montemor, M.G.S. Ferreira, N.E. Hakiki, M. Da Cunha Belo, Corros. Sci. 42 (2000) 1635

37. N.E. Hakiki, M.F. Montemor, M.G.S. Ferreira, M. Da Cunha Belo, Corros. Sci. 42 (2000) 687

38. N.E. Hakiki, M. Da Cunha Belo, J. Electrochem. Soc. 145 (1998) 3821

39. N.E. Hakiki, S. Boudin, B. Rondot, M. Da Cunha Belo, Corros. Sci. 37 (1995) 1809

40. V. Vignal, C. Valot, R. Oltra, M. Verneau, L. Coudreuse, Corros. Sci. 44 (2002) 1477

41. F. Gaben, B. Vuillemin, R. Oltra, J. Electrochem. Soc. 151 (2004) B595

42. B. Marx, “Internal report of CEST", Pr. D.D. Macdonald group, Penn State University, 2005.

43. J. Pellier, J. Geringer, B. Forest, Wear 261 (2011) 971

44. R. Greenwood, British Gas Engineering Research Report (1996) 3495M.

45. J. Carmezim, A.M. Simoes, M.F. Montemor, M. Da Cunha Belo, Corros. Sci. 47 (2005) 581

46. D. D. Macdonald and G. R. Engelhardt, ECS Transactions. 28 (2010) 123

47. D.M. Etter, J.A. Ingber, Engineering Problem Solving with $\mathrm{C}++$, Eds Prentice Hall 2nd Edition, NJ USA, 2000.

48. B.A. Forouzon, R.F. Gilberg, Computer Science: A structured Programming Approach Using C, Brooks/Cole INC, Thomson learning, Pacific Grove, Canada, 2003.

49. S.C. Cheyra, R.P. Canale, Numerical methods for engineers, WCB, Mc Graw-Hill Inc, New York, 1996. 
50. P. Combrade, in: Philippe Marcus (Ed.), Corrosion Mechanisms in Theory and Practice, Marcel Dekker Inc, New York, 2002, 349-397.

51. M. Vankeerberghen, D.D. Macdonald, Corros. Sci. 44 (2002) 1425

52. D. D. Macdonald, P. R. Wentrcek, A. C. Scott, J. Electrochem. Soc. 127(8) (1980) 1745

53. M. Wang, S. Yao, M. Madou, Sens. Actuators, B 81 (2002) 313

54. L. B. Kriksunov, D. D. Macdonald, P. J. Millett, J. Electrochem. Soc. 141(11) (1994) 3002

55. C-O.A. Olsson, M. Stemp, Electrochim. Acta 49 (2004) 2145 


\section{Figure Captions}

Figure 1: 2D image and 3D images of worn samples of Type 316L SS at applied potentials of 0 and $-200 \mathrm{mV}(\mathrm{SCE})$ after fretting corrosion for 20,000 cycles.

Figure 2: Imposed displacement $(\mu \mathrm{m})$, tangential load $(\mu \mathrm{m})$, and current $(\mu \mathrm{A})$ during 1 second of fretting at an applied potential of $-200 \mathrm{mV}$ (SCE) after 20,000 seconds of continuous fretting corrosion.

Figure 3: Schematic of the potential distribution across the barrier layer of a passive film.

Figure 4: Reactions occuring at the metal/barrier layer $(\mathrm{m} / \mathrm{bl})$ and barrier layer/solution $(\mathrm{bl} / \mathrm{s})$ interfaces, according to the Point Defect Model, resulting in the generation and annihilation of the point defects. The migration fluxes are represented by red arrows for defects that account for $\mathrm{n}$ - or $\mathrm{p}$ type conductivity in the barrier layer.

Figure 5: a) Contact zones, zone width subjected to fretting as determined by the displacement amplitude of the experiments, and the contact width between both materials; b) Increment of surface for calculating the current density and oxide layer thickness using the PDM.

Figure 6: a) Oxide layer thickness, $L_{s s}$, as a function of $p H$ and applied potential V(SHE); b) Current density, $\log$ scale, as a function of $p H$ and applied potential V(SHE), without wear, for Type 316L SS.

Figure 7: a) Oxide layer thickness, $L_{s s}$, as a function of $p H$ and applied potential V(SHE) with wear impact; b) Current density, log scale, as a function of $p H$ and applied potential V(SHE), with wear, for Type $316 \mathrm{~L}$ SS.

Figure 8: a) 2D profile of the wear zone, 316L stainless steel; simulated and actual points, applied potential of $0.25 \mathrm{~V}(\mathrm{SHE})$, experimental duration of 20,000 seconds; b) $p H$ and resistance evolution according to different zones of fretting.

Figure 9: a) Actual (black) and simulated (pink) 2D profiles wear profiles (by the PDM); applied potential of $-0.15 \mathrm{~V}(\mathrm{SHE})$, experimental duration of 20,000 seconds of continuous fretting; $b$ ) $\mathrm{pH}$ and resistance evolution for different zones of fretting.

Figure 10: a) Actual (black) and simulated (pink) 2D profiles wear profiles (by the PDM); applied potential of $0.05 \mathrm{~V}(\mathrm{SHE})$; experiments duration equal to 20,000 cycles of continuous fretting; $b$ ) $\mathrm{pH}$ and resistance evolution for different zones of fretting.

Figure 11: Experimental and simulated wear volumes as a function of applied potential after 20,000 cycles of continuous fretting.

Figure 12: Schematic illustration of the different phenomena that are postulated to be involved in the formation of the wear zone by fretting in Ringer solution. 


\section{Table Captions}

Table 1: Current density before and during fretting at different applied potentials.

Table 2: Wear depth as a function of applied potential. The Wear Depth Coefficient is the slope of the straight line of wear depth vs. fretting duration.

Table 3: Rate constant expressions: $k_{\mathrm{i}}=k_{\mathrm{i}}^{0} \mathrm{e}^{a_{i} V} \mathrm{e}^{b_{1} L} \mathrm{e}^{c_{\mathrm{i}} P H} ; \alpha_{\mathrm{i}}$ : transfer coefficient; $\gamma=F / R T, R$ : perfect gas constant, $F$ : Faraday's constant, $T$ : temperature; $k_{\mathrm{i}}^{0}$ : standard rate constant, as described by the PDM $[24,25]$.

Table 4: Standard rate constants for the interfacial defect generation/annihilation and film dissolution reactions as employed in the PDM. $k_{1}^{00}\left(\mathrm{~mol}_{\mathrm{cm}} \mathrm{cm}^{-2} \mathrm{~s}^{-1}\right)$ : base standard rate constant; $E_{a, \mathrm{j}}\left(\mathrm{kJ} \cdot \mathrm{mol}^{-1}\right)$ : Arrhenius activation energy for each reaction; $T_{0}$ : reference temperature.

Table 5: Initial parameter values implemented in the PDM.

Table 6: Medium acidification resulting from metal dissolution in a solution containing high chloride concentration. 